Groups Geom. Dyn. 6 (2012), 677-699

DOI $10.4171 / \mathrm{GGD} / 170$
Groups, Geometry, and Dynamics

(C) European Mathematical Society

\title{
A non-trivial example of a free-by-free group with the Haagerup property
}

\author{
François Gautero
}

\begin{abstract}
The aim of this note is to prove that the group of Formanek-Procesi acts properly isometrically on a finite dimensional CAT( 0 ) cube complex. This gives a first example of a nonlinear semidirect product between two non abelian free groups which satisfies the Haagerup property.
\end{abstract}

Mathematics Subject Classification (2010). 20E22, 20F65, $20 \mathrm{E} 05$.

Keywords. Haagerup property, a-T-menability, free groups, semidirect products.

\section{Introduction}

The Haagerup property is an analytical property introduced in [12], where it was proved to hold for free groups:

Definition 1 ([12], [6], [1]). A conditionally negative definite function on a discrete group $G$ is a function $f: G \rightarrow \mathbb{C}$ such that for any natural integer $n$, for any $\lambda_{1}, \ldots, \lambda_{n} \in \mathbb{C}$ with $\sum_{i=1}^{n} \lambda_{i}=0$, for any $g_{1}, \ldots, g_{n}$ in $G$ one has

$$
\sum_{i, j} \bar{\lambda}_{i} \lambda_{j} f\left(g_{i}^{-1} g_{j}\right) \leq 0
$$

The group $G$ satisfies the Haagerup property, or is an a-T-menable group, if and only if there exists a proper conditionnally negative definite function on $G$.

Groups with the Haagerup property encompass the class of amenable groups, but form a much wider class. Free groups were in some sense the "simplest" nonamenable groups with the Haagerup property. The Haagerup property has later been renewed by the work of Gromov, where it appeared under the term of a-T-menability. It is now most easily presented as a strong negation of the famous Kazhdan's property (T): in particular a group satisfies both the Haagerup and (T) properties if and only if it is a compact group (a finite group in the discrete case). We refer the reader to [6] for a detailed background and history of the Haagerup property. 
What do we know about extensions of a-T-menable groups? By [14] such an extension is a-T-menable when the quotient group is amenable. For instance, any semidirect product $\mathbb{F}_{n} \rtimes \mathbb{Z}$, where $\mathbb{F}_{n}$ denotes the rank $n$ free group, is a-T-menable. Also it has been proved recently that any wreath product $\mathbb{F}_{n} \geq \mathbb{F}_{k}$ is a-T-menable (this is a particular case of the various theorems in [8] - see also the preliminary paper [7]). But such a result does not hold anymore when considering arbitrary Haagerupby-Haagerup groups. The most famous counter-example is given by [4]: for any free subgroup $\mathbb{F}_{k}$ of $\mathrm{SL}_{2}(\mathbb{Z})$ the semidirect product $\mathbb{Z}^{2} \rtimes \mathbb{F}_{k}$ satisfies a relative version of Kazhdans's property (T) and thus is not Haagerup (see also [9] for the relative property (T) of $\mathbb{Z}^{2} \rtimes \mathrm{SL}(2, \mathbb{Z})$ and pass to a finite-index free subgroup of $\mathrm{SL}_{2}(\mathbb{Z})$; since the Haagerup property holds for a group $G$ if and only if it holds for a finite-index subgroup of $G$, this gives an example as announced).

To what extent can this result be generalized to semidirect products $\mathbb{F}_{n} \rtimes \mathbb{F}_{k}$ with both $n$ and $k$ greater or equal to 2? These semidirect products lie in some "philosophical" sense just "above" the groups $\mathbb{Z}^{2} \rtimes \mathbb{F}_{k}$ (substitute the amenable group $\mathbb{Z}^{2}$ by the free group $\mathbb{F}_{n}$, the simplest example of an a-T-menable but not amenable group) but also just above the groups $\mathbb{F}_{n} \rtimes \mathbb{Z}$ (substitute the free abelian group $\mathbb{Z}$ by the free non-abelian group $\mathbb{F}_{k}$ ). The former analogy might lead one to think that few groups $\mathbb{F}_{k} \rtimes \mathbb{F}_{n}(n, k \geq 2)$ satisfies the Haagerup property, whereas the latter one might lead one to think that any such group is an a-T-menable group. The purpose of this paper is to present a first example of a non-linear a-T-menable semidirect product $\mathbb{F}_{n} \rtimes \mathbb{F}_{k}(n, k \geq 2)$. More precisely:

Definition 2. Let $n$ be any integer greater or equal to 2. The $n^{\text {th }}$-group of FormanekProcesi is the semidirect product $\mathbb{F}_{n+1} \rtimes_{\sigma} \mathbb{F}_{n}$, where $\mathbb{F}_{n}=\left\langle t_{1}, \ldots, t_{n}\right\rangle$ and $\mathbb{F}_{n+1}=$ $\left\langle x_{1}, \ldots, x_{n}, y\right\rangle$ are the rank $n$ and rank $n+1$ free groups and $\sigma: \mathbb{F}_{n} \hookrightarrow \operatorname{Aut}\left(\mathbb{F}_{n+1}\right)$ is the monomorphism defined as follows: For $i, j \in\{1, \ldots, n\}, \sigma\left(t_{i}\right)\left(x_{j}\right)=x_{j}$ and $\sigma\left(t_{i}\right)(y)=y x_{i}$.

As claimed by this definition, it is easily checked that $\sigma$ is a monomorphism. These groups were introduced in [10] to prove that $\operatorname{Aut}\left(\mathbb{F}_{n}\right)$ is non-linear for $n \geq 3$.

Theorem 1. The $n^{\text {th }}$-group of Formanek-Procesi acts properly isometrically on some $(2 n+2)$-dimensional CAT( 0$)$ cube complex and in particular satisfies the Haagerup property.

Let us briefly recall that a cube complex is a metric polyhedral complex in which each cell is isomorphic to the Euclidean cube $[0,1]^{n}$ and the gluing maps are isometries. A cube complex is called CAT $(0)$ if the metric induced by the Euclidean metric on the cubes turns it into a CAT(0) metric space (see [3]). In order to get the above statement, we prove the existence of a "space with walls" structure as introduced by Haglund and Paulin [13]. A theorem of Chatterji-Niblo [5] or Nica [16] (for similar constructions in other settings, see also [15], [17] or [11]) gives the announced action 
on a $\operatorname{CAT}(0)$ cube complex. The referee pointed out two natural questions: Is the action on the cube complex cocompact? Is there a distortion between the distance associated to a (finite) word-metric and the wall-distance? The author thinks that the two distances should be quasi-isometric with 2 as a multiplicative constant. These questions are even more interesting in the more general context of the problem below.

Due to the profound structure theorem of [2] about subgroups of polynomially growing automorphisms (our example is a subgroup of linearly growing automorphisms), a more elaborated version of the construction presented here should hopefully lead to a positive answer to the following question:

Question (folklore). Does any semidirect product $\mathbb{F}_{n} \rtimes \mathbb{F}_{k}$ over a free subgroup of polynomially growing outer automorphisms satisfy the Haagerup property?

We guess in fact that any semidirect product $\mathbb{F}_{n} \rtimes_{\sigma} \mathbb{F}_{k}$ with $\sigma\left(\mathbb{F}_{k}\right)$ a free subgroup of unipotent polynomially growing outer automorphisms acts properly isometrically on some finite dimensional CAT(0) cube complex, the dimension of which depends on the way strata interleave with each other; see the brief discussion at the end of the paper. Since any subgroup of polynomially growing automorphisms admits a unipotent one as a finite-index subgroup [2], this would imply a positive answer to the above question.

\section{Preliminaries}

1.1. Notation. We will prove Theorem 1 with $n=2$. The reader will easily generalize the construction to any integer $n \geq 2$. With the notation of Theorem 1, the group $G:=\mathbb{F}_{3} \rtimes_{\sigma} \mathbb{F}_{2}$ admits

$$
\left\langle x_{i}, y, t_{j} \mid t_{j}^{-1} x_{i} t_{j}=x_{i}, t_{j}^{-1} y t_{j}=y x_{j}, i, j=1,2\right\rangle
$$

as a presentation. We denote by $S$ the generating set $\left\{x_{1}, x_{2}, y, t_{1}, t_{2}\right\}$ of $G$. In the structure of semidirect product $\mathbb{F}_{3} \rtimes_{\sigma} \mathbb{F}_{2}$ we will call horizontal subgroup the normal subgroup $\mathbb{F}_{3}=\left\langle x_{1}, x_{2}, y\right\rangle$ and vertical subgroup the subgroup $\mathbb{F}_{2}=\left\langle t_{1}, t_{2}\right\rangle$. Any element is uniquely written as a concatenation $t w$ where $t$ is a vertical element, i.e., an element in the vertical subgroup, and $w$ is a horizontal element, i.e., an element in the horizontal subgroup. We denote by $\mathcal{A}$ the alphabet over $S \cup S^{-1}$ and by $\pi$ the map which, to a given word in $\mathcal{A}$, assigns the unique element of $G$ that it defines. A reduced word is a word without any cancellation $x x^{-1}$ or $x^{-1} x$. Words consisting of vertical (resp. horizontal) letters are vertical (resp. horizontal) words. A reduced representative of an element $g$ in $G$ is a reduced word in the alphabet $\mathcal{A}$ whose image under $\pi$ is $g$.

We denote by $\Gamma$ the Cayley graph of $G$ with respect to $S$. Since the vertices of $\Gamma$ are in bijection with the elements of $G$, we do not distinguish between a vertex of $\Gamma$ and the element of $G$ associated to this vertex. The edges of $\Gamma$ are oriented: an edge 
of $\Gamma$ is denoted by the pair "(initial vertex of the edge, terminal vertex of the edge)". The edges are labeled with the elements in $S \cup S^{-1}$. For instance, the edge $\left(g, g x_{i}\right)$ has label $x_{i}$, whereas the edge $\left(g x_{i}, g\right)$ has label $x_{i}^{-1}$. If $x$ is the label of an edge we will term this edge $x$-edge. If $E$ is an oriented edge, then $E^{-1}$ is the same edge with the opposite orientation. For instance, $\left(g, g t_{i}\right)^{-1}=\left(g t_{i}, g\right)$. A reduced edge-path in $\Gamma$ is an edge-path which reads a reduced word. When considering $\Gamma$ as a cellular complex, there is exactly one 1-cell associated to the two edges $(g, g s)(s \in S)$ and $(g s, g)$, and each orientation of this 1-cell corresponds to one of these edges.

Lemma 1.1. With the notation above, the group $G$ admits $S_{\min }:=\left\{y, t_{1}, t_{2}\right\} \subset S$ as a generating set.

Proof. For $i \in\{1,2\}$ we have $x_{i}=y^{-1} t_{i}^{-1} y t_{i}$, hence the lemma.

A straightforward consequence is

Corollary 1.2. Let $\chi_{i}$ be the set of 1-cells of $\Gamma$ associated to edges with label $x_{i}^{ \pm 1}$, and let $\Gamma_{c}$ be the closure of the complement of $\chi_{1} \cup \chi_{2}$ in $\Gamma$. Then $\Gamma_{c}$ is ( $G$-equivariantly homeomorphic to) the Cayley graph of $G$ with respect to the generating set $S_{\min }$ defined in Lemma 1.1 .

1.2. Space with walls structure. Spaces with walls were introduced in [13] in order to check the Haagerup property. A space with walls is a pair $(X, W)$ where $X$ is a set and $\mathcal{W}$ is a family of partitions of $X$ into two classes, called walls, such that for any two distinct points $x, y$ in $X$ the number of walls $\omega(x, y)$ is finite. This is the wall distance between $x$ and $y$. We say that a discrete group acts properly on a space with walls $(X, \mathcal{W})$ if it leaves invariant $\mathcal{W}$ and for some (and hence any) $x \in X$ the function $g \mapsto \omega(x, g x)$ is proper on $G$.

Theorem 1.3 ([13]). A discrete group $G$ which acts properly on a space with walls satisfies the Haagerup property.

In order to get Theorem 1 we will need the (stronger) result below (we refer to [16] for a similar statement). Let $(X, W)$ be a space with walls. Say that two walls $\left(u, u^{\mathrm{c}}\right) \in \mathcal{W}$ and $\left(v, v^{\mathrm{c}}\right) \in \mathcal{W}$ cross if all four intersections $u \cap v, u \cap v^{\mathrm{c}}, u^{\mathrm{c}} \cap v$ and $u^{\mathrm{c}} \cap v^{\mathrm{c}}$ are non-empty. We denote by $I(\mathcal{W})$ the (possibly infinite) supremum of the cardinalities of finite collections of walls which pairwise cross.

Theorem 1.4 ([5]). Let $G$ be a discrete group which acts properly on a space with walls $(X, \mathcal{W})$. Then $G$ acts properly isometrically on some I(W)-dimensional $\mathrm{CAT}(0)$ cube complex. In particular it satisfies the Haagerup property. 


\section{Horizontal and vertical walls}

\subsection{Definition and stabilizers}

Definition 2.1. The horizontal block $\mathcal{Y}$ is the set of all the elements in $G$ which admit $t y w$, with $t$ a vertical word and $w$ a horizontal word, as a reduced representative. A horizontal wall is a left-translate $g\left(\mathcal{Y}, y^{\mathrm{c}}\right), g \in G$.

The vertical $j$-block $\mathcal{V}_{j}$ is the set of all the elements in $G$ which admit $t_{j} t w$, with $t$ a vertical word and $w$ a horizontal word, as a reduced representative. A vertical $j$-wall is a left-translate $g\left(\mathcal{V}_{j}, \mathcal{V}_{j}^{c}\right), g \in G$.

See Figure 1 for an illustration of the horizontal walls. By definition of a reduced representative, in the definition of $\mathcal{Y}$ (resp. of $\mathcal{V}_{j}$ ), w (resp. $t$ ) does not begin with $y^{-1}$ (resp. with $t_{j}^{-1}$ ).

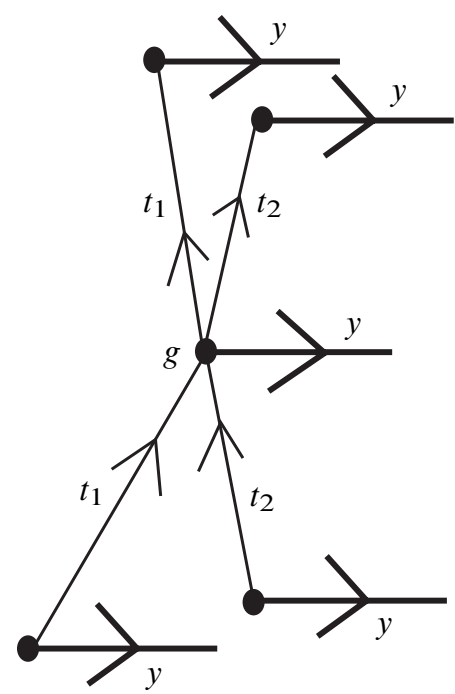

Figure 1. Horizontal wall.

Lemma 2.2. The collection of all the horizontal walls is $G$-invariant for the leftaction of $G$ on itself. The same assertion is true for the collection of all the vertical walls. Moreover:

(1) The left G-stabilizer of any horizontal wall is a conjugate of the vertical subgroup.

(2) The horizontal subgroup is both the left and right $G$-stabilizer of any vertical wall. 
Proof. By definition the collection of either all the horizontal or all the vertical walls consists of all the left $G$-translates of the horizontal or vertical walls $\left(\mathcal{Y}, \mathcal{y}^{\mathrm{c}}\right)$ or $\left(\mathcal{V}_{j}, \mathcal{V}_{j}^{c}\right)$ so that it is invariant under the left $G$-action.

Let $g \in y$. Then $g=t y w$ for some $t$ in the vertical subgroup and $w$ in the horizontal one. If $t^{\prime}$ is another element in the vertical subgroup, $t^{\prime} g=t^{\prime} t y w \in X_{i}$. Thus the vertical subgroup is in the $G$-stabilizer of $\mathcal{Y}$. By the relation $u t=t \sigma(t)(u)$ for $u$ in the horizontal subgroup (recall that $\sigma: \mathbb{F}_{2} \hookrightarrow \operatorname{Aut}\left(\mathbb{F}_{3}\right)$ is the monomorphism such that $G=\mathbb{F}_{3} \rtimes_{\sigma} \mathbb{F}_{2}$ ) we get $u g=t \sigma(t)(u) y w$ so that $\langle u\rangle$ does not stabilize $\mathcal{Y}$. Since any element of $G$ is the concatenation of a vertical element with a horizontal one, these observations imply that the $G$-stabilizer of $y$ is the vertical subgroup. Since the horizontal walls are left $G$-translates of the wall $\left(\mathcal{y}, y^{c}\right)$, the $G$-stabilizer of a horizontal wall is a conjugate of the vertical subgroup.

The proof for the stabilizers of the vertical walls is similar and easier: just observe that since the horizontal subgroup is normal in $G$, it is useless to take its conjugates.

\subsection{Finiteness of horizontal and vertical walls between any two elements}

Proposition 2.3. There are a finite number of vertical walls between any two elements.

Proof. The vertical walls are the usual walls used to prove that the free group $\mathbb{F}_{2}$ satisfies the Haagerup property. Thus there are a finite number (in fact one) of vertical walls between $g \in G$ and $g t_{i}$ with $t_{i}$ a vertical generator. By Lemma 2.2, each vertical wall is stabilized by the right-action of the horizontal subgroup. Thus no vertical wall separates $g$ from $g s, g \in G$ and $s$ a horizontal generator $x_{i}$ or $y$. The proposition follows.

Proposition 2.4. There are a finite number of horizontal walls between any two elements in $G$.

This proposition is a little harder than the previous one, and we need a preliminary lemma:

Lemma 2.5. Each side of each horizontal wall is invariant under the right-action of the vertical subgroup: if $\left(\mathscr{H}, \mathscr{H}^{\mathrm{c}}\right)$ is an horizontal wall, then for any element $t$ of the vertical subgroup we have $\mathscr{H} t=\mathscr{H}$ and $\mathscr{H}^{\mathrm{c}} t=\mathscr{H}^{\mathrm{c}}$. In particular, each horizontal wall is invariant under the right-action of the vertical subgroup.

Proof. We begin with the proof of the following assertion.

Claim 1. Let $Y$ be the intersection of $y$ with the horizontal subgroup $\mathbb{F}_{3}$. Then $Y$ is invariant under the conjugation-action of the vertical subgroup $\mathbb{F}_{2}$.

Proof. Let $w \in Y$. We write the reduced word $w=y m_{0} y^{\epsilon_{1}} m_{1} \ldots y^{\epsilon_{i}} m_{i} \ldots y^{\epsilon_{k}} m_{k}$, $k \geq 0, \epsilon_{i} \in\{ \pm 1\}$, with $m_{i}$ a reduced group word in the letters $x_{1}^{ \pm 1}, x_{2}^{ \pm 1}$ (since $w$ 
is reduced for each $i$ satisfying $\epsilon_{i}+\epsilon_{i+1}=0$, we have $m_{i} \neq 1$ ). Then $t_{1}^{-1} w t_{1}=$ $y \mu_{0} y^{\epsilon_{1}} \mu_{1} \ldots y^{\epsilon_{i}} \mu_{i} \ldots y^{\epsilon_{k}} \mu_{k}$, where $\mu_{i}$ has the form $a_{i} m_{i} b_{i}^{-1}$ with

$a_{i}=x_{1}$ if $\epsilon_{i}=1$ and $a_{i}=1$ otherwise,

$b_{i}=x_{1}^{-1}$ if $\epsilon_{i+1}=-1$ and $b_{i}=1$ otherwise,

for $i=1, \ldots, k$ and setting $\epsilon_{k+1}=1$.

Therefore, if $i<k$ and $\epsilon_{i}+\epsilon_{i+1}=0$, then $\mu_{i}$ is conjugate to $m_{i}$ so that it is non trivial. Whenever $\epsilon_{i}+\epsilon_{i+1} \neq 0$, no cancellation might occur after reduction between $y^{\epsilon_{i}}$ and $y^{\epsilon_{i+1}}$ even if $\mu_{i}$ is reduced to the trivial word. Thus, after writing the $\mu_{i}$ as reduced words, the word $y \mu_{0} y^{\epsilon_{1}} \mu_{1} \ldots y^{\epsilon_{k}} \mu_{k}$ we eventually get is reduced, so that $t_{1}^{-1} w t_{1} Y \subset Y$. The reverse inclusion being analogous we have $t_{1}^{-1} w t_{1} Y=Y$ and similarly $t_{2}^{-1} w t_{2} Y=Y$.

Now $y=\bigcup_{t \in \mathbb{F}_{2}} t Y=\bigcup_{t \in \mathbb{F}_{2}}\left(t Y t^{-1}\right) t$, which by the claim is equal to $\bigcup_{t \in \mathbb{F}_{2}} Y t$. Therefore, $u y_{t}=u \mathcal{y}$ for any $t$ in the vertical subgroup and for any $u$ in the horizontal one. Each one of the previous equalities holds when substituting $y^{c}$ for $y$ so that in particular $u\left(\mathcal{Y}, y^{c}\right) t=u\left(\mathcal{y}, y^{c}\right)$ for any $t$ in the vertical subgroup and for any $u$ in the horizontal one. Lemma 2.5 is proved.

Proof of Proposition 2.4. By Lemma 2.5, there is no horizontal wall between any two elements $g$ and $g t_{j}, j=1,2$. On the other hand we have the following claim:

Claim 2. For any $g \in G, \bigcup_{t \in \mathbb{F}_{2}}(g t, g t y)^{ \pm 1}$ disconnects $\Gamma_{c}$ in two connected components, which are the two sides of the horizontal wall $g\left(\mathcal{Y}, y^{c}\right)$.

Thus there is exactly one horizontal wall between any two elements $g$ and $g y$. By Lemma 1.1, we get the finiteness of the number of horizontal walls between any two elements in $G$.

\section{Vertizontal walls}

Before beginning, we recall that $\Gamma_{c}$ denotes the Cayley graph of $G$ with respect to $S_{\min }=\left\{y, t_{1}, t_{2}\right\}$ (see Lemma 1.1). We also recall that in a Cayley graph, $\left(g, g t_{i}\right)$ denotes the edge with label $t_{i}$ (a $t_{i}$-edge) oriented from $g$ to $g t_{i}$ and $\left(g t_{i}, g\right)$ the same edge with the opposite orientation (its label is thus $t_{i}^{-1}$, it is a $t_{i}^{-1}$-edge). Finally, if $E$ is an oriented edge, then $E^{-1}$ denotes the same edge with the opposite orientation. In particular $\left(g, g t_{i}\right)^{-1}=\left(g t_{i}, g\right)$.

\subsection{Definition and stabilizers}

Definition 3.1. With the notation above: let $H_{i}:=\left\langle x_{i+1}, t_{i+1}, y x_{i} y^{-1} t_{i}, x_{i} t_{i}^{-1}\right\rangle$ $(i=1,2 \bmod 2)$, let $E_{i}^{+}:=H_{i}\left(e, t_{i}\right), E_{i}^{-}:=H_{i}\left(t_{i}, e\right)$ and $E_{i}:=E_{i}^{+} \cup E_{i}^{-}$.

The $i$-block $\mathcal{T}_{i}$ is the set of all the elements in $G$ which are connected to the identity vertex $e$ by an edge-path in $\Gamma_{c} \backslash E_{i}$. 
See Figures 2 and 3. Beware that Figure 2 might be slightly misleading when considering the edge-paths $y x_{i} y^{-1}$ : they are indeed preserved under the right-action of $t_{i}$ but this is a consequence of the fact that a cancellation occurs between $\sigma\left(t_{i}\right)\left(x_{i}\right)=$ $x_{i}$ and $\sigma\left(t_{i}\right)\left(y^{-1}\right)=x_{i}^{-1} y^{-1}$. Since we did not draw the images of the edges in these edge-paths, this cancellation does not appear in the figure.

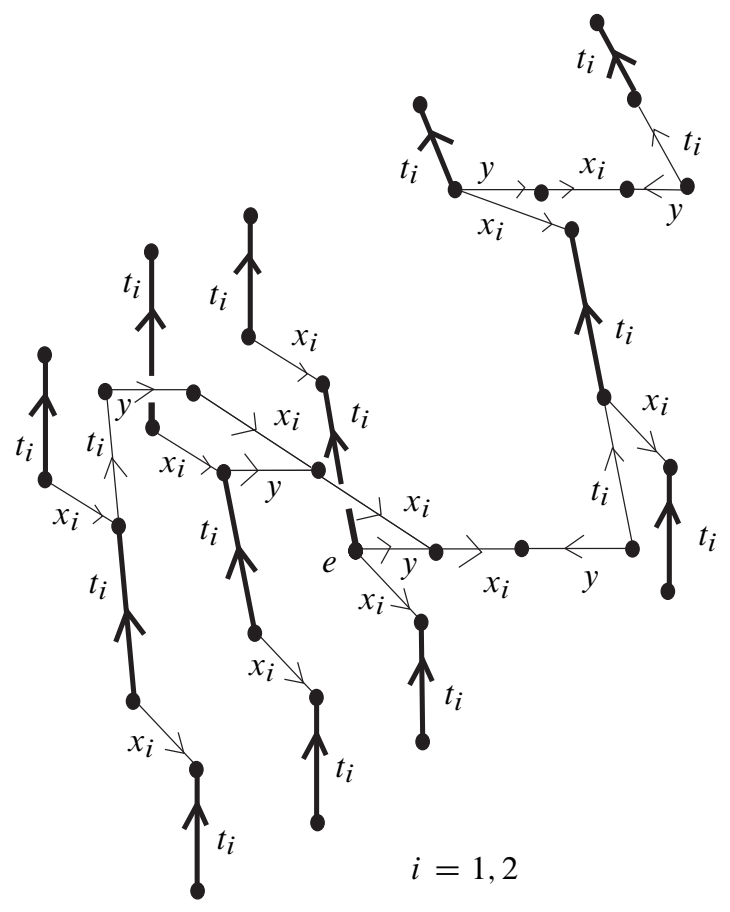

Figure 2. Some edges in $E_{i}$.

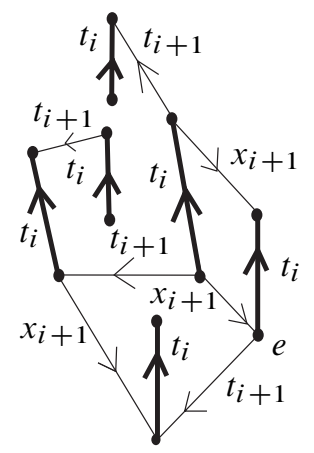

Figure 3. Other edges in $E_{i}$. 
Remark 3.2. Observe that $x_{i+1}=y^{-1} t_{i+1}^{-1} y t_{i+1}, y x_{i} y^{-1} t_{i}=y t_{i} y^{-1}$ and $x_{i} t_{i}^{-1}=$ $y^{-1} t_{i}^{-1} y$, i.e., $H_{i}=\left\langle y^{-1} t_{i+1}^{-1} y t_{i+1}, t_{i+1}, y t_{i} y^{-1}, y^{-1} t_{i} y\right\rangle$. In particular, for any $k \in \mathbb{Z}, y t_{i}^{k} y^{-1}$ and $y^{-1} t_{i}^{k} y$ are in $H_{i}$. See Figure 4 .

Lemma 3.3. All the initial (resp. terminal) vertices of the edges in $E_{i}^{+}$are connected to e (resp. to $\left.t_{i}\right)$ by an edge-path in $\Gamma_{c} \backslash E_{i}$.

Proof. The edge-path $\left(e, y^{-1}\right)\left(y^{-1}, y^{-1} t_{i}^{-1}\right)\left(y^{-1} t_{i}^{-1}, y^{-1} t_{i}^{-1} y\right)$ connects $e$ to $x_{i} t_{i}^{-1}$ in $\Gamma_{c} \backslash E_{i}$. The edge-path $(e, y)\left(y, y t_{i}\right)\left(y t_{i}, y t_{i} y^{-1}\right)$ connects $e$ to $t_{i} y x_{i} y^{-1}=$ $y x_{i} y^{-1} t_{i}$ in $\Gamma_{c} \backslash E_{i}$. The edge $\left(e, t_{i+1}\right)$ (indices are written modulo 2) connects $e$ to $t_{i+1}$ in $\Gamma_{c} \backslash E_{i}$. The edge-path $\left(e, t_{i+1}^{-1}\right)\left(t_{i+1}^{-1}, t_{i+1}^{-1} y\right)\left(t_{i+1}^{-1} y, t_{i+1}^{-1} y t_{i+1}\right)\left(t_{i+1}^{-1} y t_{i+1}\right.$, $\left.t_{i+1}^{-1} y t_{i+1} y^{-1}\right)$ connects $e$ to $x_{i+1}$.

Since $E_{i}$ is left $H_{i}$-invariant, taking and concatenating the left-translates of the previous edge-paths by elements of $H_{i}$ we connect all the initial vertices of the edges in $\left\langle x_{i+1}, t_{i+1}, y x_{i} y^{-1} t_{i}, x_{i} t_{i}^{-1}\right\rangle\left(e, t_{i}\right)=E_{i}$ by edge-paths in $\Gamma_{c} \backslash E_{i}$.

For connecting $t_{i}$ to $x_{i}$ (resp. $t_{i}$ to $y x_{i} y^{-1} t_{i}^{2}, t_{i}$ to $x_{i+1} t_{i}$ ) in $\Gamma_{c} \backslash E_{i}$ just take the left-translate by $t_{i}$ of the edge-path between $e$ and $x_{i} t_{i}^{-1}$ (resp. between $e$ and $t_{i} y x_{i} y^{-1}$, between $e$ and $\left.x_{i+1}\right)$ : indeed recall that $t_{i} x_{i}=x_{i} t_{i}, t_{i} x_{i+1}=x_{i+1} t_{i}$ and $t_{i} y x_{i} y^{-1}=y x_{i} y^{-1} t_{i}$. For connecting $t_{i}$ to $t_{i+1} t_{i}$ : first connect $t_{i}$ to $x_{i}$ by the edge-path given above, then $x_{i}$ to $x_{i} t_{i+1}$ by the edge $\left(x_{i}, x_{i} t_{i+1}\right)$, then $x_{i} t_{i+1}$ to $x_{i} t_{i+1} t_{i} x_{i}^{-1}=t_{i+1} t_{i}$ by the left $t_{i+1}$-translate of the edge-path between $t_{i}$ and $x_{i}$. We conclude as for the initial vertices.

Corollary 3.4. Any element in $G$ is connected either to e or to $t_{i}$ by an edge-path in $\Gamma_{c} \backslash E_{i}$.

Proof. Let $g \in G$ and consider any edge-path $p$ in $\Gamma_{c}$ from (the vertex of $\Gamma_{c}$ associated to) $g$ to $e$. If $p \subset \Gamma_{c} \backslash E_{i}$ we are done. Otherwise $p$ passes through some edge in $E_{i}$, we denote by $q$ the subpath of $p$ from $g$ to the initial vertex $v$ of the first edge of $E_{i}$ in $p$. By Lemma 3.3, the initial (resp. terminal) vertex of each edge in $E_{i}^{+}$is connected to $e$ (resp. to $t_{i}$ ) in $\Gamma_{c} \backslash E_{i}$. Thus there is an edge-path $r$ in $\Gamma_{c} \backslash E_{i}$ from $v$ to either $e$ or $t_{i}$. The concatenation $q r$ gives an edge-path in $\Gamma_{c} \backslash E_{i}$ from $g$ to either $e$ or $t_{i}$.

In what follows, in order to have a more readable text we will write each edge-path as a concatenation of the labels of its edges: in order to ensure that this defines an edge-path in $\Gamma_{c} \backslash E_{i}$ the reader will have each time to remind the starting-point of the edge-path.

Lemma 3.5. No edge-path in $\Gamma_{c} \backslash E_{i}$ containing only $y^{ \pm 1}$-edges and $t_{i}^{ \pm 1}$-edges connects e to $t_{i}$.

Since the proof below is rather long, we first give an idea of what happens: the only way to go from $e$ to $x_{i}$ or $t_{i}$ without going through an $x_{i}^{ \pm 1}$-edge (which does not 
exist in $\left.\Gamma_{c}\right)$ is to go through $t_{i}^{ \pm 1}$-edges appearing in the relations $\left(y^{-1} t_{i}^{-1} y\right) t_{i}=x_{i}$ or $\left(y t_{i} y^{-1}\right) t_{i}\left(y t_{i}^{-1} y^{-1}\right)=t_{i}$ or their inverses (in parentheses the generators of $H_{i}$ involved - the $t_{i}^{ \pm 1}$-edges in $E_{i}$ are those outside the parentheses). As the reader can check (see Figure 4), the edges in $E_{i}$ have been chosen to "cut" these relations. Each time one goes through a $y^{ \pm 1}$-edge, one crosses a horizontal wall, and since $e$ and $t_{i}$ are in the same side of any horizontal wall (Claim 3), one has to cross it back, and in particular when crossing $(g, g y)$ one has to go back to the right vertical orbit of $g$, i.e., to some $g t, t \in \mathbb{F}_{2}$ (Claim 6). If this is not a $H_{i}$-translate of the right vertical orbit of the identity of $G$, then one has a vertical short-cut from $g$ to $g t$. In this way, by an induction process, we prove that reduced edge-paths in $\Gamma_{c} \backslash E_{i}$ going from $e$ to $t_{i}$, which minimize the number of horizontal edges crossed, are concatenations of subpaths of the form $t_{i}^{k} y t_{i}^{l} y^{-1}$ (at least when assuming that one began with a $y$-edge as first horizontal edge - see Claim 9). Claims 10 and 11 rely upon the fact that left-translating an edge-path in $\Gamma_{c} \backslash E_{i}$ by an element in $H_{i}$ yields an edge-path in $\Gamma_{c} \backslash E_{i}$ : each subpath $y t_{i}^{l} y^{-1}$ defines such an element. Hence any subpath of $p$ remains in the same side of $E_{i}$ as $e$, hence the contradiction (Claim 12). By Claim 13, if the first horizontal edge in $p$ is a $y^{-1}$-edge, then there exists a reduced edge-path in $\Gamma_{c} \backslash E_{i}$ from $e$ to $t_{i}$ whose first horizontal edge is a $y$-edge and minimizing the number of horizontal edges it crosses, hence the conclusion (Claim 13 is proved by arguments similar to those exposed above).

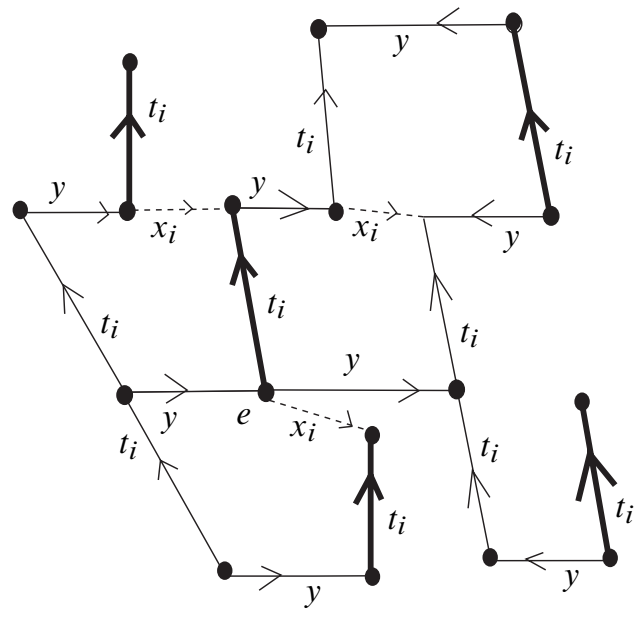

$i=1,2$

Figure 4

Proof. We begin with an easy assertion about horizontal walls:

Claim 3. The vertices $e$ and $t_{i}$ are in the same side of any horizontal wall $g\left(y, y^{c}\right)$. 
Proof. By Lemma 2.5, each side of each horizontal wall is invariant under the rightaction of the vertical subgroup. Of course $e$ and $t_{i}=e t_{i}$ belong to the same orbit for the right-action of the vertical subgroup hence the claim.

Now an easy assertion about Definition 3.1:

Claim 4. Let $\phi: G \rightarrow \mathbb{Z}$ be the morphism defined by $\phi(y)=1$ and $\phi\left(t_{j}\right)=0$ for $j=1,2$ (since the sum of the exponents of the letters $y$ in the relators of $G$ is zero, this morphism is well defined and since the elements $y, t_{1}, t_{2}$ generate $G$ - see Lemma 1.1 - it is defined over the whole group $G)$. If $\left(g, g t_{i}\right)^{ \pm 1}$ is any edge in $E_{i}$, then for any vertical element $t \in \mathbb{F}_{2}$ the element $g t$ belongs to $\operatorname{Ker}(\phi)$.

Proof. Definition 3.1 gives $E_{i}=H_{i}\left(e, t_{i}\right)^{ \pm 1}, H_{i}:=\left\langle x_{i+1}, t_{i+1}, y x_{i} y^{-1} t_{i}, x_{i} t_{i}^{-1}\right\rangle$. Each element in the subgroup $H_{i}$ belongs to $\operatorname{Ker}(\phi)$ since this is true for each generator. The claim follows from the fact that $e$ belongs to $\operatorname{Ker}(\phi)$ and all the elements in the vertical orbit of an element in $\operatorname{Ker}(\phi)$ also are in $\operatorname{Ker}(\phi)$ since $\phi\left(t_{j}\right)=0$ for $j=1,2$.

Let $\mathcal{P}_{e, t_{i}}$ be the set of all the reduced edge-paths in $\Gamma_{c} \backslash E_{i}$ from $e$ to $t_{i}$ passing only through $y^{ \pm 1}$ - and $t_{i}^{ \pm 1}$-edges. Let $\mathcal{P}_{e, t_{i}}^{\min } \subset \mathcal{P}_{e, t_{i}}$ be the subset formed by all the edge-paths in $\mathcal{P}_{e, t_{i}}$ minimizing the number of horizontal edges they cross. Let $p \in \mathcal{P}_{e, t_{i}}^{\min }$.

Claim 5. There is at least one horizontal edge in $p$ and this $y$ - or $y^{-1}$-edge begins at the vertical orbit of $e$.

Proof. The set of all the vertical edges between vertices in a same vertical right-orbit forms a tree (a copy of the Cayley graph of $\mathbb{F}_{2}$ ). Of course the vertices $e$ and $t_{i}$ belong to the same vertical right-orbit. The non-existence of a vertical edge-path between $e$ and $t_{i}$ then follows from the fact that $\left(e, t_{i}\right)^{ \pm 1}$ belongs to $E_{i}$. The conclusion follows.

Claim 6. Assume that $p$ goes through the $y$-edge $(g, g y), g \in G$. Then there is $k \in \mathbb{Z}$ such that $p$ goes through the $y^{-1}$-edge $\left(g t_{i}^{k} y, g t_{i}^{k}\right)$.

Proof. By definition $p$ goes from $e$ to $t_{i}$. By Claim 3, $e$ and $t_{i}$ are in the same side of any horizontal wall. By Claim 2, if $p$ goes through the $y$-edge $(g, g y)$, then it changes side in the horizontal wall $g\left(\mathcal{Y}, y^{c}\right)$ and has to cross back some $y$-edge with initial vertex in the orbit of $g$ under the right action of the vertical subgroup. This is exactly Claim 6.

Claim 7. Assume that the first horizontal edge in $p$ is a $y$-edge. Then $p$ admits an initial subpath of the form $t_{i}^{k_{0}} y t_{i}^{k_{1}} y^{-1}\left(k_{0}\right.$ might be zero). 
Proof. Assume that $p$ does not satisfy the announced property. Then the second horizontal edge in $p$ also is a $y$-edge. Let $\left(\mathscr{H}, \mathscr{H}^{c}\right)$ be the horizontal wall associated to this $y$-edge. By Claim 3,e and $t_{i}$ lie in the same side of $\left(\mathscr{H}, \mathscr{H}^{c}\right)$. Thus $p$ crosses back $\left(\mathscr{H}, \mathscr{H}^{c}\right)$. By Claim 2 and since we assumed that $p$ does not cross any $t_{i+1}^{ \pm 1}$-edge (indices are written modulo 2), there is a non-trivial subpath $q_{1}$ of $p=q_{0} q_{1} q_{2}$ starting at the initial vertex $g$ of the above $y$-edge and going back to some $g t_{i}^{m}(m \in \mathbb{Z})$. But the initial subpath of $p$ leading to $g$ is written as $t_{i}^{k_{0}} y t_{i}^{k_{1}}$. The element in $G$ that it defines does not belong to $\operatorname{Ker}(\phi)$, see Claim 4 . By this last claim no edge between two vertices in the right orbit of $g$ under the vertical subgroup belongs to $E_{i}$. Hence the vertical edge-path $r$ between $g$ and $g t_{i}^{m}$ is an edge-path in $\Gamma_{c} \backslash E_{i}$ : it has the same endpoints as $q_{1} \subset p=q_{0} q_{1} q_{2}$ and crosses at least one horizontal edge less than $q$ since $q_{1}$ is a reduced edge-path starting with a $y$-edge. Therefore the concatenation $q_{0} r q_{2}$, possibly after reduction, defines an edge-path in $\mathcal{P}_{e, t_{i}}$ which crosses at least one horizontal edge less than $p$ : this is a contradiction with $p \in \mathcal{P}_{\boldsymbol{e}, t_{i}}^{\min }$.

Claim 8. Assume that $p$ admits an initial subpath $q_{0}$ of the form $t_{i}^{k_{0}} y t_{i}^{k_{1}} y^{-1} \ldots$ $t_{i}^{k_{2 j}} y t_{i}^{k_{2 j+1}} y^{-1}\left(k_{i} \neq 0\right.$ for $\left.i>0\right)$. If the first horizontal edge following $q_{0}$ in $p$ is a $y$-edge, then $p$ admits an initial subpath of the form $q_{0} t_{i}^{k_{2 j+2}} y t_{i}^{k_{2 j+3}} y^{-1}$.

Proof. This amounts to proving that the second horizontal edge following $q_{0}$ is a $y^{-1}$-edge. The argument for proving this claim is exactly the same as for Claim 7: if the second horizontal edge following $q_{0}$ in $p$ also were a $y$-edge, then the vertical edge-path from its initial vertex $g$ to $g t_{i}^{m}$ is in $\Gamma_{c} \backslash E_{i}$ : indeed the edge-path in $p$ from $e$ to $g$ reads $t_{i}^{k_{0}} y t_{i}^{k_{1}} y^{-1} \ldots t_{i}^{k_{2 j}} y t_{i}^{k_{2 j+1}} y^{-1} t^{k_{2 j+2}} y$ and so is not in $\operatorname{Ker}(\phi)$ (see Claim 4). The conclusion is as in Claim 7.

Claim 9. Assume that the first horizontal edge in $p$ is a $y$-edge. Then $p$ is the reduced concatenation of edge-paths reading words of the form $t_{i}^{k_{2 j}} y t_{i}^{k_{2 j+1}} y^{-1}$ in $\Gamma_{c} \backslash E_{i}$.

Proof. By Claim 7, $p$ admits a non-trivial initial subpath reading $t_{i}^{k_{0}} y t_{i}^{k_{1}} y^{-1}$. By Claim 8, it suffices to prove that the horizontal edge in $p$ following this initial subpath is not a $y^{-1}$-edge. Assume that it is, i.e., $p=t_{i}^{k_{0}} y t_{i}^{k_{1}} y^{-1} t_{i}^{k_{2}} y^{-1} \ldots$ By Claim 6, the edge-path $q_{1}=t_{i}^{k_{2}} y^{-1} \ldots$ following $q_{0}=t_{i}^{k_{0}} y t_{i}^{k_{1}} y^{-1}$ in $p$ has to go back to some $g t_{i}^{n}$, if $g$ is the terminal vertex of $q_{0}$ (hence the initial vertex of a $y$-edge). Since $p \in \mathcal{P}_{e, t_{i}}^{\min }$, there is an edge in $E_{i}^{+}$between $g$ and $g t_{i}^{n}$. Since $t_{i}^{k_{m}} y t_{i}^{k_{m+1}} y^{-1}=$ $y x_{i}^{k_{m+1}} y^{-1} t_{i}^{k_{m+1}} t_{i}^{k_{m}} \in H_{i} t_{i}^{k_{m}}$, the edge-path $q_{0}$ reads an element of the form $h t_{i}^{n}$ with $h \in H_{i}$. By left-translation by $h^{-1}$ we pull-back $q_{1}$ to an edge-path starting at $e$ and ending at some $t_{i}^{l}$ with $l>0$ since there is an edge in $E_{i}^{+}$between the initial and terminal vertex. Since there are at least two horizontal edges in $q_{0}$, we so get an edge-path in $\mathcal{P}_{e, t_{i}}$ from $e$ to $t_{i}$ which has less horizontal edges than $p$. This contradicts $p \in \mathcal{P}_{\boldsymbol{e}, t_{i}}^{\min }$. 
Claim 10. Assume that $p$ is the reduced concatenation of edge-paths reading words of the form $t_{i}^{k_{2 j}} y t_{i}^{k_{2 j+1}} y^{-1}$ in $\Gamma_{c} \backslash E_{i}$ for $j$ from 0 to $l$. Then $\sum_{j=0}^{l} k_{2 j} \leq 0$.

Proof. We proceed by induction on $l$. For $l=0$ we have $p=t_{i}^{k_{0}} y t_{i}^{k_{1}} y^{-1}$. Since $p$ starts at $e$ and $\left(e, t_{i}\right) \in E_{i}$, we necessarily have $k_{0} \leq 0$. Let us assume that the claim holds at $l$ and let us prove that it then holds at $l+1$. We observe that for any non-negative integer $j$ the element $y t_{i}^{k_{2 j+1}} y^{-1}$ is in $H_{i}$. Thus a left-translate by (the inverse of) such an element of an edge-path $q$ is in $\Gamma_{c} \backslash E_{i}$ if and only if $q$ already was. We first left-translate the edge-path reading $t_{i}^{k_{2}} y \ldots$ and starting at $t_{i}^{k_{0}} y t_{i}^{k_{1}} y^{-1}$ by $\left(y t_{i}^{k_{1}} y^{-1}\right)^{-1}$ : we get an edge-path starting at $e$, reading $t_{i}^{k_{0}} t_{i}^{k_{2}} y t_{i}^{k_{3}} y^{-1} \ldots$ and lying in $\Gamma_{c} \backslash E_{i}$ since $p$ is in $\Gamma_{c} \backslash E_{i}$. Since $\left(e, t_{i}\right) \in E_{i}$, this implies $k_{0}+k_{2} \leq 0$. We continue the process by left-translating by $\left(y t_{i}^{k_{3}} y^{-1}\right)^{-1}$ the subpath of $p$ starting with $t_{i}^{k_{4}} y$, and more generally by $\left(y t_{i}^{k_{2 j+1}} y^{-1}\right)^{-1}$ the subpath of $p$ starting with $t_{i}^{k_{2 j+2}} y$. We eventually get $k_{0}+k_{2}+\cdots+k_{2 l} \leq 0$ and the claim is proved.

Claim 11. Let $g=\prod_{j=0}^{l} t_{i}^{k_{2 j}} y t_{i}^{k_{2 j+1}} y^{-1}$ be an element in $G$. Let $\phi_{i}: G \rightarrow \mathbb{Z}$ be the map which to an element $g$ assigns the sum of the exponents of the letters $x_{i}$ appearing in the unique reduced representative of $g$ of the form $w t$ where $w$ is a reduced horizontal word and $t$ is a reduced vertical one (beware that $\phi_{i}$ is not a morphism since its values on certain relators is non-zero). Then $\phi_{i}(g)=\sum_{j=0}^{l} k_{2 j+1}$.

Proof. We prove by induction on $l$ that writing $g \in G$ with the generating set $S$ yields the expression

$$
g=y x_{i}^{k_{1}+k_{3}+\cdots+k_{2 l+1}} y^{-1} t_{i}^{k_{0}+k_{1}+\cdots+k_{2 l}+k_{2 l+1}} .
$$

If $l=0$ then $g=t_{i}^{k_{0}} y t_{i}^{k_{1}} y^{-1}=y x_{i}^{-k_{0}} t_{i}^{k_{0}+k_{1}} y^{-1}=y x^{-k_{0}} x_{i}^{k_{0}+k_{1}} y^{-1} t_{i}^{k_{0}+k_{1}}=$ $y x_{i}^{k_{1}} y^{-1} t_{i}^{k_{0}+k_{1}}$. So the assertion holds for $l=0$. Assume that it holds for $l$. Then if $g=\prod_{j=0}^{l+1} t_{i}^{k_{2 j}} y t_{i}^{k_{2 j+1}} y^{-1}=\left(\prod_{j=0}^{l} t_{i}^{k_{2 j}} y t_{i}^{k_{2 j+1}} y^{-1}\right)\left(t_{i}^{k_{2 l+2}} y t_{i}^{2 k_{2 l+3}} y^{-1}\right)$ by induction hypothesis we get the equality

$$
g=\left(y x_{i}^{k_{1}+k_{3}+\cdots+k_{2 l+1}} y^{-1} t_{i}^{k_{0}+k_{1}+\cdots+k_{2 l+1}}\right)\left(t_{i}^{k_{2 l+2}} y t_{i}^{k_{2 l+3}} y^{-1}\right) .
$$

Hence, by permuting $t_{i}^{k_{0}+k_{1}+\cdots+k_{2 l+1}+k_{2 l+2}}$ with $y$ using the relation $t_{i} y=y x_{i}^{-1} t_{i}$ (notice that the exponent of $x_{i}$ is the opposite of the exponent of $t_{i}$ ), we obtain the following writing of $g$ :

$$
g=y x_{i}^{k_{1}+k_{3}+\cdots+k_{2 l+1}} y^{-1} y x_{i}^{-k_{0}-k_{1}-\cdots-k_{2 l+2}} t_{i}^{k_{0}+k_{1}+\cdots+k_{2 l+1}+k_{2 l+2}+k_{2 l+3}} y^{-1} .
$$

This is easier rewritten as follows:

$$
g=y x_{i}^{-k_{0}-k_{2}-\cdots-k_{2 l+2}} t_{i}^{k_{0}+k_{1}+\cdots+k_{2 l+1}+k_{2 l+2}+k_{2 l+3}} y^{-1} .
$$


By permuting $t_{i}^{k_{0}+k_{1}+\cdots+k_{2 l+1}+k_{2 l+2}+k_{2 l+3}}$ with $y^{-1}$ using the relation $t_{i} y^{-1}=$ $x_{i} y^{-1} t_{i}$ (notice that the exponent of $x_{i}$ is equal to the exponent of $t_{i}$ ), this gives the formula

$$
\begin{gathered}
g=y x_{i}^{-k_{0}-k_{2}-\cdots-k_{2 l+2}} x_{i}^{k_{0}+k_{1}+\cdots+k_{2 l+1}+k_{2 l+2}+k_{2 l+3}} \\
y^{-1} t_{i}^{k_{0}+k_{1}+\cdots+k_{2 l+1}+k_{2 l+2}+k_{2 l+3}} .
\end{gathered}
$$

Another rewriting gives the easier expression

$$
g=y x_{i}^{k_{1}+k_{3}+\cdots+k_{2 l+1}+k_{2 l+3}} y^{-1} t_{i}^{k_{0}+k_{1}+\cdots+k_{2 l+1}+k_{2 l+2}+k_{2 l+3}},
$$

and the induction is complete. Since $\phi_{i}(g)$ is equal to the sum of the exponents of the $x_{i}$ in the previous writing, we get the claim.

Claim 12. The first horizontal edge in $p$ is not a $y$-edge.

Proof. We argue by contradiction and assume that the first horizontal edge in $p$ is a $y$-edge. By Claim 9, $p$ is the reduced concatenation of edge-paths reading words of the form $t_{i}^{k_{2 j}} y t_{i}^{k_{2 j+1}} y^{-1}$ in $\Gamma_{c} \backslash E_{i}$ for $j$ from 0 to $l(l \geq 0)$. By Claim 10, $\sum_{j=0}^{l} k_{2 j} \leq 0$. Since the element defined by $p$ is $t_{i}$ and the exponent of $t_{i}$ in $p$ is $\sum_{j=0}^{l} k_{2 j}+\sum_{j=0}^{l} k_{2 j+1}$, we have $\sum_{j=0}^{l} k_{2 j}+\sum_{j=0}^{l} k_{2 j+1}=1$. Hence $\sum_{j=0}^{l} k_{2 j+1}>0$. Claim 11 then gives $\phi_{i}\left(t_{i}\right)>0$, which is an absurdity since $\phi_{i}\left(t_{i}\right)=0$, hence the claim.

Claim 13. If there is $p \subset \mathcal{P}_{e, t_{i}}^{\min }$ admitting a $y^{-1}$-edge as first horizontal edge, then there is $q \subset \mathcal{P}_{e, t_{i}}^{\min }$ admitting a $y$-edge as first horizontal edge.

Proof. The arguments are similar to those exposed above for proving that $p$ does not begin with a $y$-edge. Assume that the first horizontal edge in $p$ is a $y^{-1}$-edge, i.e., $p=t_{i}^{k_{0}} y^{-1} \ldots$ with $k_{0} \leq 0$. If $\phi$ is the morphism given in Claim $4, \phi\left(t^{k_{0}} y^{-1}\right)=-1$ so that, by this same Claim 4 , there is no edge in $E_{i}$ between two vertices in the orbit of the terminal vertex of this $y^{-1}$-edge under the vertical subgroup. By Claim $6, p$ has to cross back the associated horizontal wall. Moreover, the number of horizontal edges in $p$ is minimal. Therefore this $y^{-1}$-edge is followed by an edge-path of the form $t_{i}^{k_{1}} y$ in $\Gamma_{c} \backslash E_{i}$, i.e., $p=q_{0} q_{1}$ with $q_{0}=t_{i}^{k_{0}} y^{-1} t_{i}^{k_{1}} y$ and $q_{1} \subset \Gamma_{c} \backslash E_{i}$. If the first horizontal edge in $q_{1}$ is also a $y^{-1}$-edge we repeat the argument. Thus we eventually get a non-trivial reduced edge-path $p^{\prime}$, the first horizontal edge of which is a $y$-edge such that $p=t_{i}^{k_{0}} y^{-1} t_{i}^{k_{1}} y \ldots t_{i}^{k_{2 m}} y^{-1} t_{i}^{k_{2 m+1}} y p^{\prime}$.

We noticed above that $k_{0} \leq 0$. From Remark 3.2, $y^{-1} t_{i}^{k_{2 j+1}} y \in H_{i}$. Hence for any integer $j$ from 0 to $m$ the left-translate of the subpath $t_{i}^{k_{2 j+2}} y^{-1} \ldots$ by $\left(y^{-1} t_{i}^{k_{2 j+1}} y\right)^{-1}$ yields an edge-path in $\Gamma_{c} \backslash E_{i}$. We eventually get $\sum_{j=0}^{m} k_{2 j} \leq 0$ 
(the same construction and argument have been exposed with more details in the proof of Claim 10). In $G$ we have

$$
t_{i}^{k_{0}} y^{-1} t_{i}^{k_{1}} y \ldots t_{i}^{k_{2 m}} y^{-1} t_{i}^{k_{2 m+1}} y=x_{i}^{-k_{1}-k_{3}-\cdots-k_{2 m+1}} t_{i}^{k_{0}+k_{1}+\cdots+k_{2 m}+k_{2 m+1}}:=g
$$

so that $\phi(g) \leq-\phi_{i}\left(x_{i}\right)\left(\phi_{i}\right.$ is the map from $G$ onto $\mathbb{Z}$ giving the exponent of $x_{i}$; see Claim 11). So the terminal vertex of any edge-path $t_{i}^{k_{0}} y^{-1} t_{i}^{k_{1}} y \ldots t_{i}^{k_{2 m}} y^{-1} t_{i}^{k_{2 m+1}} y$ starting at $e$ lies in the same side as $e$ in the grid $\left\langle x_{i}, t_{i}\right\rangle$.

Thus there is $r \in \mathbb{N}$ such that $t_{i}^{k_{0}} y^{-1} t_{i}^{k_{1}} y \ldots t_{i}^{k_{m}} y^{-1} t_{i}^{k_{m+1}} y t_{i}^{r}$ ends at some power of $x_{i}^{-1} t_{i}$, i.e., as a group element defines a power $\left(x_{i}^{-1} t_{i}\right)^{s}$ with $s \in \mathbb{Z}$. The left-translate of the edge-path $t_{i}^{k_{0}} y^{-1} t_{i}^{k_{1}} y \ldots t_{i}^{k_{m}} y^{-1} t_{i}^{k_{m+1}} y t_{i}^{r}\left(t_{i}^{-r} p^{\prime}\right)$ by $t_{i}^{-r} y^{-1} t_{i}^{-k_{m+1}} y t_{i}^{-k_{m}} y^{-1} \ldots y^{-1} t^{-k_{1}} y t^{-k_{0}}$ yields, after reduction, an edge-path in $\Gamma_{c} \backslash E_{i}$ from $e$ to $\left(x_{i}^{-1} t_{i}\right)^{-s} t_{i}$. Since $\left(x_{i}^{-1} t_{i}\right)^{-s} t_{i}=t_{i}\left(x_{i}^{-1} t_{i}\right)^{-s}$, by post-composing it with an edge-path reading $y^{-1} t_{i}^{-s} y$ if $s>0$ and $y^{-1} t_{i}^{s} y$ if $s<0$ we get an edgepath $q$ in $\Gamma_{c} \backslash E_{i}$ which belongs to $\mathcal{P}_{e, t_{i}}^{\min }$ (it has at most the same number of horizontal edges as $p$ ), and the first horizontal edge of which is a $y$-edge since $p^{\prime}$ begins with a $y$-edge.

If there exists an edge-path between $e$ and $t_{i}$ in $\Gamma_{c} \backslash E_{i}$ which goes only through horizontal and $t_{i}^{ \pm 1}$-edges, then there exists such an edge-path $p$ which is reduced and minimizes the number of horizontal edges that it crosses. By Claim 5, such an edge-path $p$ contains at least one horizontal edge. By Claim 12, the first horizontal edge in $p$ is not a $y$-edge. It follows by Claim 13, that the first horizontal edge in $p$ neither is a $y^{-1}$-edge. We so eventually get that there exists no edge-path in $\Gamma_{c} \backslash E_{i}$ from $e$ to $t_{i}$ and Lemma 3.5 is proved.

Lemma 3.6. If there exists an edge-path connecting $e$ to $t_{i}$ in $\Gamma_{c} \backslash E_{i}$, then there exists an edge-path composed only of horizontal edges and of $t_{i}^{ \pm 1}$-edges connecting e to $t_{i}$ in $\Gamma_{c} \backslash E_{i}$.

Proof. Assume the existence of an edge-path $p$ in $\Gamma_{c} \backslash E_{i}$ from $e$ to $t_{i}$. Then $p=$ $w_{0} w_{1} \ldots w_{2 k}$ where

(1) $w_{2 j}$ is an edge-path passing only through horizontal and $t_{i}^{ \pm 1}$-edges,

(2) $w_{2 j-1}$ is an edge-path defining an element in the subgroup $\left\langle x_{i+1}, t_{i+1}\right\rangle$ and does not pass through any $t_{i}^{ \pm 1}$-edges.

Since $\left\langle x_{i+1}, t_{i+1}\right\rangle \subset H_{i}$, by a left-translation of $w_{2} \ldots w_{2 k}$ by $w_{1}^{-1}$ we get an edge-path $w_{2}^{1} \ldots w_{2 k}^{1}$ in $\Gamma_{c} \backslash E_{i}$ starting at the initial vertex of $w_{1}$ and ending at $w_{1}^{-1} t_{i}$. Thus the concatenation $w_{0} w_{2}^{1} \ldots w_{2 k}^{1}$ defines an edge-path in $\Gamma_{c} \backslash E_{i}$ from $e$ to $w_{1}^{-1} t_{i}$. By repeating this process we eventually get an edge-path $q=w_{0} w_{2}^{1} \ldots w_{2 k}^{k}$ in $\Gamma_{c} \backslash E_{i}$ from $e$ to $w_{2 k-1}^{-1} \ldots w_{1}^{-1} t_{i}$ where $w_{2 j}^{j}$ passes only through horizontal and $t_{i}^{ \pm 1}$-edges whereas $w_{2 k-1}^{-1} \ldots w_{1}^{-1}$ is an element in $\left\langle x_{i+1}, t_{i+1}\right\rangle$. Since $q$ starts at $e$ 
and passes only through horizontal and $t_{i}^{ \pm 1}$-edges, its terminal vertex is an element $g$ in $\left\langle y, t_{i}\right\rangle$. Let $h \in\left\langle x_{i+1}, t_{i+1}\right\rangle$ with $g=h t_{i}$. Then $h=g t_{i}^{-1}$ so that $h \in\left\langle y, t_{i}\right\rangle$ since both $g$ and $t_{i}$ belong to $\left\langle y, t_{i}\right\rangle$. Therefore $h \in\left\langle x_{i+1}, t_{i+1}\right\rangle \cap\left\langle y, t_{i}\right\rangle=\{e\}$. It follows that $q$ ends at $t_{i}$ so that $q$ is an edge-path as announced.

Corollary 3.7. There are exactly two connected components in $\Gamma_{c} \backslash E_{i}$ : the connected component of $e$ and the connected component of $t_{i}$.

Proof. By Lemmas 3.5 and 3.6, $e$ and $t_{i}$ lie in two distinct connected components of $\Gamma_{c} \backslash E_{i}$. By Corollary 3.4, these are the only two connected components of $\Gamma_{c} \backslash E_{i}$.

By Corollary 3.7, if $\mathcal{T}_{i}$ denotes an $i$-block (see Definition 3.1), then $\left(\mathcal{T}_{i}, \mathcal{T}_{i}^{\mathrm{c}}\right)$ is a wall so that the following definition makes sense:

Definition 3.8. A vertizontal $i$-wall $(i=1,2)$ is any left-translate $g\left(\mathcal{T}_{i}, \mathcal{T}_{i}^{\mathrm{c}}\right), g \in G$, of an $i$-block $\mathcal{T}_{i}$ (see Definition 3.1).

Lemma 3.9. The collection of all the vertizontal $i$-walls $(i=1,2)$ is $G$-invariant for the left-action of $G$ on itself. The left $G$-stabilizer of any vertizontal $i$-wall $(i=1,2 \bmod 2)$ is a conjugate of the subgroup $H_{i}=\left\langle x_{i+1}, t_{i+1}, y x_{i} y^{-1} t_{i}, x_{i} t_{i}^{-1}\right\rangle$.

Proof. The left $G$-invariance is obvious, as in the proof of Lemma 2.2. Let us check the assertion about the left $G$-stabilizers. A vertizontal wall is a left $G$-translate of $\left(\mathcal{T}_{i}, \mathcal{T}_{i}^{\mathrm{c}}\right)$, where $\mathcal{T}_{i}$ is an $i$-block, see Definition 3.1. Thus its left $G$-stabilizer is conjugate in $G$ to the left $G$-stabilizer of $\left(\mathcal{T}_{i}, \widetilde{\mathcal{T}}_{i}^{\mathrm{c}}\right)$. Since $\mathcal{T}_{i}$ is separated from $\widetilde{\mathcal{T}}_{i}^{\mathrm{c}}$ by the left $H_{i}$-translates of $\left(e, t_{i}\right)^{ \pm 1}$ (see Definition 3.1), this left $G$-stabilizer is $H_{i}$.

\subsection{Finiteness of the number of vertizontal walls between any two elements}

Proposition 3.10. There are a finite number of vertizontal walls between any two elements in $G$.

Proof. We consider the set of vertizontal 1-walls (the proof is the same for the vertizontal 2-walls). We work with the generating set $S_{\min }=\left\{y, t_{1}, t_{2}\right\}$ given by Lemma 1.1. Since any $y^{ \pm 1}$ - and any $t_{2}^{ \pm 1}$-edge lies in $\Gamma_{c} \backslash\left(\bigcup_{g \in G} g E_{1}\right)$ (see Definition 3.1), no vertizontal 1-wall is intersected when passing from $g$ to $g y$ nor from $g$ to $g t_{2}$ whatever $g \in G$ is considered. Thus one only has to check which vertizontal 1-walls are intersected when passing from $e$ to $t_{1}$. There is of course the wall $\left(\mathcal{T}_{1}, \mathcal{T}_{1}^{\mathrm{c}}\right)$. Assume that there is another wall $g\left(\mathcal{T}_{1}, \mathcal{T}_{1}^{\mathrm{c}}\right)$. Then, by definition, this wall corresponds to the partition of $\Gamma_{c}$ in two components given by $g E_{1}$. Thus $\left(e, t_{1}\right)^{ \pm 1} \in g E_{1}$. Let $a \in E_{1}$ with $\left(e, t_{1}\right)^{ \pm 1}=g a$. By definition of $E_{1}$ there is $h \in H_{1}$ (see Definition 3.1) with $a=h\left(e, t_{1}\right)^{ \pm 1}$ hence $\left(e, t_{1}\right)^{ \pm 1}=g h\left(e, t_{1}\right)^{ \pm 1}$. 
Since the stabilizer of any 1 -cell is trivial, we get $g=h^{-1}$ so that $g E_{1}=E_{1}$. This implies that $g\left(\mathcal{T}_{1}, \widetilde{\mathcal{T}}_{1}^{\mathrm{c}}\right)=\left(\mathcal{T}_{1}, \mathcal{T}_{1}^{\mathrm{c}}\right)$, and we are done.

\section{A proper action}

Although obvious, the following proposition is indispensable:

Proposition 4.1. The set of all the horizontal, vertical and vertizontal walls defines a space with walls structure $(G, W)$ for $G$. The left action of $G$ on itself defines an action on this space with walls structure.

Proof. By Propositions 2.3, 2.4 and 3.10, there are a finite number of walls between any two elements so that $(G, \mathcal{W})$ is a space with walls structure.

We now prove the following result.

Proposition 4.2. The action of $G$ on the space with walls structure $(G, W)$ given by Proposition 4.1 is proper.

Proof. Before beginning let us recall that what is important is the algebraic intersection-number of the edge-paths with each wall: if a given path $p$ intersects two times a wall $\left(W, W^{\mathrm{c}}\right)$ first passing from $W$ to $W^{\mathrm{c}}$, then crossing back from $W^{\mathrm{c}}$ to $W$, this intersection-number is zero.

We work with the classical generating set $S=\left\{x_{1}, x_{2}, y, t_{1}, t_{2}\right\}$ of $G$. Each element $g \in G$ admits an unique reduced representative of the form $w t$ with $w$ a reduced horizontal word and $t$ a reduced vertical word. Since the vertical walls are the classical walls in the free group $\mathbb{F}_{2}$, the number of vertical walls intersected goes to infinity with the number of letters in $t$. Thus we can assume that $g$ admits the reduced horizontal word $w$ as a reduced representative.

Recall that the intersections of the horizontal walls with the horizontal subgroup give classical walls of the free group. By Lemma 2.5, horizontal walls are invariant under the right-action of the vertical subgroup. In particular any two $y^{ \pm 1}$-edge in the reduced horizontal word $w$ define distinct horizontal walls. It follows that the number of horizontal walls intersected goes to infinity with the number of $y^{ \pm 1}$-letters in $w$. Thus we can assume that $w$ contains only $x_{i}^{ \pm 1}$-letters, $i=1$, 2 i.e. we can assume that $w=x_{i_{1}}^{k_{1}} x_{i_{2}}^{k_{2}} \ldots x_{i_{r}}^{k_{r}}$ with $k_{j} \in \mathbb{Z}$ and $i_{j} \in\{1,2\}, i_{j} \neq i_{j+1}$.

Two vertizontal $i$-walls separate $e$ from $x_{i}$ in $\Gamma_{c}$ : the $i$-wall $\left(\mathcal{T}_{i}, \mathcal{T}_{i}^{\mathrm{c}}\right)$ and the $i$-wall $y^{-1}\left(\mathcal{T}_{i}, \mathcal{T}_{i}^{\mathrm{c}}\right)$. These are indeed the two walls intersected exactly once by the edge-path starting at $e$ and reading $y^{-1} t_{i}^{-1} y t_{i}$. Of course they also separate $e$ from $x_{i}^{k}(k \in \mathbb{Z})$. The left-translates by $h \in G$ of these two $i$-walls separate $h$ from $h x_{i}^{k}$. This readily implies that there are at least two vertizontal walls intersected by any edge-path $x_{i_{j}}^{k_{j}}$ in $w$. Moreover, the two $i$-walls given previously for passing from 
$e$ to $x_{i}^{k}$ are necessarily distinct from those given for passing from $x_{i}^{k} x_{j}^{l}(j \neq i)$ to $x_{i}^{k} x_{j}^{l} x_{i}^{m}(k, l, m \in \mathbb{Z})$ : indeed $x_{i}^{k} x_{j}^{l}(i \neq j)$ does not belong to the stabilizer of a vertizontal $i$-wall. We so found a collection of $i$-walls intersected by the $x_{i_{j}}^{k_{j}}$ in $w$ which are all distinct and whose number goes to infinity with the number of times the letters $x_{1}^{ \pm 1}$ and $x_{2}^{ \pm 1}$ alternate in $w$ (since the number of intersections is increased by 2 each times one reads a new word of the form $x_{i}^{k}, i=1$ or $i=2$ ). Therefore we can assume that $w=x_{1}^{k}$ with $k \in \mathbb{N}$.

The $2 k$ left-translates by $x_{1}, x_{1}^{2}, \ldots, x_{1}^{k-1}$ of the vertizontal 1 -walls $\left(\mathcal{T}_{1}, \mathcal{T}_{1}^{\mathrm{c}}\right)$ and $y^{-1}\left(\mathcal{T}_{1}, \mathcal{T}_{1}^{\mathrm{c}}\right)$ separate $e$ from $x_{1}^{k}$ : these are indeed the walls crossed exactly once by the edge-path in $\Gamma_{c}$ starting at $e$, ending at $x_{1}^{k}$ and reading $y^{-1} t_{1}^{-k} y t_{1}^{k}$. We thus get the proposition.

\section{The Haagerup property and dimension of the cube complex}

We give here, as corollaries of the construction developed above, the two main results we were interested in: the Haagerup property for $G$ and the, stronger, fact that $G$ acts properly isometrically on a cube complex (Theorem 1).

Corollary 5.1. The group $G$ satisfies the Haagerup property.

Proof. By Propositions 4.1 and 4.2, $G$ acts properly on a space with walls structure $(G, W)$. By [13], $G$ satisfies the Haagerup property.

Corollary 5.2. The group $G$ acts properly isometrically on some 6-dimensional cube complex, where 6 is the supremum of the cardinalities of collections of walls which pairwise cross in the space with walls structure for $G$ given by Proposition 4.1.

Proof. Let $(G, \mathcal{W})$ be the space with walls structure for $G$ given by Proposition 4.1. Let us recall that $\mathcal{W}$ is the set of all the horizontal, vertical and vertizontal walls. By Proposition 4.2, $G$ acts properly on $(G, \mathcal{W})$. By [5], $G$ acts properly isometrically on some $I(\mathcal{W})$-dimensional cube complex where $I(\mathcal{W})$ is the supremum of the cardinalities of collections of walls which pairwise cross (see Theorem 1.4).

Lemma 5.3. With the notation above: let $\mathcal{F}$ be a collection of walls in $(G, \mathcal{W})$ which pairwise cross. Then there is at most one vertical wall and one horizontal wall in $\mathcal{F}$.

Proof. Vertical walls are the classical walls of the free group: the two sides of such a wall are separated by an edge of the Cayley graph with respect to a basis of the free group (a tree). Thus two distinct such walls satisfy that one of the two sides of a wall properly contains a side of the other. Consequently, two distinct vertical walls do not pairwise cross. Let us now consider two distinct horizontal walls. By Claim 2, the two sides of a horizontal wall are separated by $\bigcup_{t \in \mathbb{F}_{2}}(g t, g t y)^{ \pm 1}$. Thus, as it is the 
case for the free group, one of the two sides of a wall properly contains a side of the other. Lemma 5.3 is proved.

Lemma 5.4. With the notation of Lemma 5.3, for each $i \in\{1,2\}$ :

(1) The vertizontal $i$-walls $\left(\mathcal{T}_{i}, \mathcal{T}_{i}^{\mathrm{c}}\right)$ and $y\left(\mathcal{T}_{i}, \mathcal{T}_{i}^{\mathrm{c}}\right)$ cross.

(2) There are at most two vertizontal $i$-wall in $\mathcal{F}$.

Proof. The following claim is obvious:

Claim 14. For any $g \in G$ either $g E_{i} \cap E_{i}=\emptyset$, which is equivalent to $g \notin H_{i}$, or $g E_{i}=E_{i}$, which is equivalent to $g \in H_{i}$.

Claim 15 below is extracted from the proof of Lemma 3.3.

Claim 15. Let $g_{0}, g_{1}$ be the two initial (resp. terminal) vertices of an edge in $g E_{i}^{+}$, $g \notin H_{i}$ (we recall that $E_{i}=E_{i}^{+} \cup E_{i}^{-}$with $E_{i}^{+}=H_{i}\left(e, t_{i}\right)$ ). Then there is a reduced edge-path in $\Gamma_{c} \backslash g E_{i}$ between $g_{0}$ and $g_{1}$ satisfying the following properties:

- It is a concatenation of edge-paths of four kinds: edge-path reading words of the form $\left(y t_{i} y^{-1}\right)^{ \pm 1}$, edge-paths reading words of the form $\left(y^{-1} t_{i} y\right)^{ \pm 1}$ and edgepaths reading words of the form $t_{i+1}^{ \pm 1}$ or $\left(y^{-1} t_{i+1}^{-1} y t_{i+1}\right)^{ \pm 1}(i=1,2 \bmod 2)$.

- Both the initial and terminal vertices of each of the above subpaths are the initial (resp. terminal) vertices of $t_{i}$-edges in $g E_{i}^{+}$.

Assume that two distinct vertizontal $i$-walls $g_{1}\left(\mathcal{T}_{i}, \mathcal{T}_{i}^{\mathrm{c}}\right)$ and $g_{2}\left(\mathcal{T}_{i}, \mathcal{T}_{i}^{\mathrm{c}}\right)$ cross. Then (just apply a left-translation by $\left.g_{1}^{-1}\right)\left(\mathcal{T}_{i}, \mathcal{T}_{i}^{\mathrm{c}}\right)$ and $g\left(\mathcal{T}_{i}, \mathcal{T}_{i}^{\mathrm{c}}\right)$ cross, with $g=g_{1}^{-1} g_{2}$. It is thus sufficient to prove that there is at most one left-coset $g H_{i}\left(g \notin H_{i}\right)$ such that $\left(\widetilde{T}_{i}, \mathcal{T}_{i}^{\mathrm{c}}\right)$ and $g\left(\widetilde{\mathcal{T}}_{i}, \mathcal{T}_{i}^{\mathrm{c}}\right)$ cross.

There is a reduced edge-path $p$ in $\Gamma_{c} \backslash\left(E_{i} \cup g E_{i}\right)$ between $e$ and the initial vertex $g_{0}$ of some edge in $g E_{i}$. Without loss of generality assume that $g_{0} \in g \widetilde{T}_{i}$, which is equivalent to $\left(g_{0}, g_{0} t_{i}\right) \in g E_{i}^{+}$. Then $\left\{e, g_{0}\right\} \subset \mathcal{T}_{i} \cap g \mathcal{T}_{i}$ so that in particular $\widetilde{\tau_{i}} \cap g{\widetilde{\tau_{i}}}^{\prime} \neq \emptyset$.

By Claim 14, since $\left(e, t_{i}\right) \in E_{i}$ (resp. $\left(g_{0}, g_{0} t_{i}\right) \in g E_{i}$ and $\left.g \notin H_{i}\right)$, we have $\left(e, t_{i}\right) \notin g E_{i}$ (resp. $\left.\left(g_{0}, g_{0} t_{i}\right) \notin E_{i}\right)$. Therefore, setting $q=p\left(g_{0}, g_{0} t_{i}\right)$ we get a reduced edge-path $q \subset \Gamma_{c} \backslash E_{i}$ between $e$ and $g_{0} t_{i}$ so that $g_{0} t_{i} \in \mathcal{T}_{i}$. Moreover, since $\left(g_{0}, g_{0} t_{i}\right) \in g E_{i}^{+}, g_{0} t_{i} \in g{\mathcal{\mathcal { T } _ { i }}}^{\mathrm{c}}$. Hence $g_{0} t_{i} \in \mathcal{T}_{i} \cap g \mathcal{T}_{i}^{\mathrm{c}}$ so that $\mathcal{T}_{i} \cap g{\widetilde{\mathcal{T}_{i}}}^{\mathrm{c}} \neq \emptyset$. Similarly, setting $r=p^{-1}\left(e, t_{i}\right)$ we get an edge-path in $\Gamma_{c} \backslash g E_{i}$ so that $t_{i} \in g \mathcal{T}_{i}$. Since $t_{i} \in \mathcal{T}_{i}^{\mathrm{c}}$, this implies that $g \widetilde{\mathcal{T}}_{i} \cap \mathcal{T}_{i}^{\mathrm{c}} \neq \emptyset$.

At this point we thus proved that $\mathcal{T}_{i} \cap g \mathcal{T}_{i}, \widetilde{T}_{i} \cap g \mathcal{T}_{i}^{\mathrm{c}} \neq \emptyset$ and $g \mathcal{T}_{i} \cap \mathcal{T}_{i}^{\mathrm{c}} \neq \varnothing$ (of course, if we had assumed $g_{0} \in g{\widetilde{\mathcal{T}_{i}}}^{\mathrm{c}}$ instead of $g_{0} \in g \mathcal{T}_{i}$, we would also have found three non-empty intersections among the four possible intersections between the different sides of the walls; however they would not have been the same but

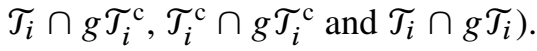

Assume now $\mathcal{T}_{i}^{\mathrm{c}} \cap g \mathcal{T}_{i}^{\mathrm{c}} \neq \emptyset$. Since $t_{i} \in \mathcal{T}_{i}^{\mathrm{c}}$, there exists a reduced edge-path $s$ in $\Gamma_{c} \backslash E_{i}$ from $t_{i}$ to some element in $g \mathcal{T}_{i}^{\mathrm{c}}$. Since $t_{i} \in g \mathcal{T}_{i}$, this edge-path $s$ crosses an edge $\left(g_{1}, g_{1} t_{i}\right)$ in $g E_{i}^{+}$, and we can assume that it crosses only one such edge. 
Let us denote by $q^{\prime}$ the subpath of $s$ from $t_{i}$ to $g_{1}: q^{\prime}$ is a reduced edge-path in $\Gamma_{c} \backslash\left(E_{i} \cup g E_{i}\right)$. Let us consider a reduced edge-path $q^{\prime \prime}$ in $\Gamma_{c} \backslash g E_{i}$ from $g_{1}$ to $g_{0}$ as given by Claim 15. Assume that $q^{\prime \prime}$ is contained in $\Gamma_{c} \backslash E_{i}$. Then $q^{\prime} q^{\prime \prime} p$ is an edge-path in $\Gamma_{c} \backslash E_{i}$ from $e$ to $t_{i}$, which is impossible. Therefore $q^{\prime \prime}$ crosses an edge in $E_{i}$. But, by construction (see Claim 15), the only $t_{i}^{ \pm 1}$-edges crossed by $q^{\prime \prime}$ belong to subpaths of the form $\left(y t_{i} y^{-1}\right)^{ \pm 1}$ or $\left(y^{-1} t_{i} y\right)^{ \pm 1}$ and the initial and terminal vertices of these subpaths are in $g E_{i}$. Thus these $t_{i}^{ \pm 1}$-edges crossed by $q^{\prime \prime}$ are $t_{i}^{ \pm 1}$-edges in $y g E_{i}$ or in $y^{-1} g E_{i}$. Since they belong to $y g E_{i} \cap E_{i}$ or to $y g E_{i} \cap E_{i}$, by Claim 14, we get $y g \in H_{i}$ or $y^{-1} g \in H_{i}$. Hence $g \in y H_{i}$ or $g \in y^{-1} H_{i}$. From all which

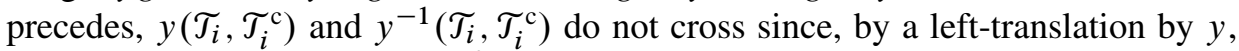

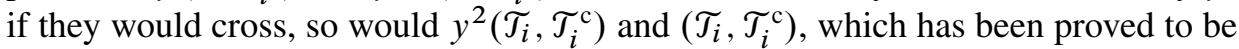
false. We so got that if two vertizontal $i$-walls $\mathcal{Z}$ and $\mathcal{Z}^{\prime}$ cross, then there is $g \in G$ such

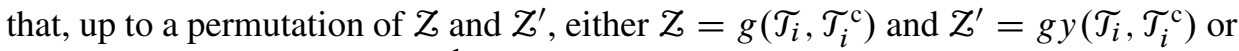
$\mathcal{Z}=g\left(\widetilde{T}_{i}, \widetilde{T}_{i}^{c}\right)$ and $Z^{\prime}=g y^{-1}\left(\mathcal{T}_{i}, \widetilde{\mathcal{T}}_{i}^{\mathrm{c}}\right)$. This implies item (2) of Lemma 5.4.

It only remains to check that $\left(\widetilde{T}_{i},{\widetilde{\mathcal{T}_{i}}}^{\mathrm{c}}\right)$ and $y\left(\mathcal{T}_{i},{\widetilde{\mathcal{T}_{i}}}^{\mathrm{c}}\right)$ cross. Obviously (use the

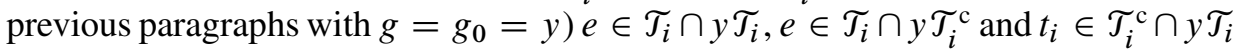

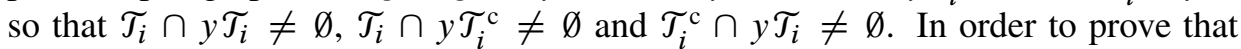
${\widetilde{T_{i}}}^{\mathrm{c}} \cap y{\widetilde{T_{i}}}^{\mathrm{c}} \neq \emptyset$, let us observe that $t_{i} \in \mathcal{T}_{i}^{\mathrm{c}}$ is connected to $y t_{i} x_{i}^{-1} t_{i}$ by the edgepath $\left(t_{i}, t_{i} y\right)\left(t_{i} y, t_{i} y t_{i}\right)$ since $t_{i} y=y x_{i}^{-1} t_{i}$ and $t_{i} x_{i}^{-1}=x_{i}^{-1} t_{i}$. The edge $\left(t_{i}, t_{i} y\right)$ is a $y$-edge so belongs to $\Gamma_{c} \backslash E_{i}$. The edge $\left(t_{i} y, t_{i} y t_{i}\right)=\left(y t_{i} x_{i}^{-1}, y t_{i} x_{i}^{-1} t_{i}\right)$ is in $y E_{i}$ so, by Claim 14, does not belong to $E_{i}$. Hence $\left(t_{i}, t_{i} y\right)\left(t_{i} y, t_{i} y t_{i}\right)$ is an edge-path in $\Gamma_{c} \backslash E_{i}$ from $t_{i} \in \mathcal{T}_{i}^{\mathrm{c}}$ to $y t_{i} x_{i}^{-1} t_{i}$, so that $y t_{i} x_{i}^{-1} t_{i} \in \mathcal{T}_{i}^{\mathrm{c}}$. Moreover, we have that $y t_{i} \in y \mathcal{T}_{i}^{\mathrm{c}}$ is connected to $y t_{i} x_{i}^{-1} t_{i}=y t_{i} y^{-1} t_{i} y$ by the edge-path $\left(y t_{i}, y t_{i} y^{-1}\right)\left(y t_{i} y^{-1}, y t_{i} y^{-1} t_{i}\right)\left(y t_{i} y^{-1} t_{i}, y t_{i} y^{-1} t_{i} y\right)$. The first and last edge in this edge-path are respectively $y^{-1}$ - and $y$-edges and so belong to $\Gamma_{c} \backslash y E_{i}$. The $t_{i}$-edge $\left(y t_{i} y^{-1}, y t_{i} y^{-1} t_{i}\right)$ is in $E_{i}$ since $y t_{i} y^{-1} \in H_{i}$, see Remark 3.2. By Claim 14, it is not in $y E_{i}$. We thus proved that $\left(y t_{i}, y t_{i} y^{-1}\right)\left(y t_{i} y^{-1}, y t_{i} y^{-1} t_{i}\right)\left(y t_{i} y^{-1} t_{i}, y t_{i} y^{-1} t_{i} y\right)$ is an edge-path from $y t_{i} \in y{\widetilde{\mathcal{T}_{i}}}^{\mathrm{c}}$ to $y t_{i} x_{i}^{-1} t_{i}$ in $\Gamma_{c} \backslash y E_{i}$ so that $y t_{i} x_{i}^{-1} t_{i} \in y{\widetilde{\mathcal{T}_{i}}}^{\mathrm{c}}$. Now $y t_{i} x_{i}^{-1} t_{i} \in{\mathcal{\tau _ { i }}}^{\mathrm{c}}$ and $y t_{i} x_{i}^{-1} t_{i} \in y{\widetilde{\mathcal{T}_{i}}}^{\mathrm{c}}$ so that ${\widetilde{\mathcal{T}_{i}}}^{\mathrm{c}} \cap y{\mathcal{\mathcal { T } _ { i }}}^{\mathrm{c}} \neq \emptyset$, and item (1) of Lemma 5.4 is proved.

Let us now conclude the proof of Corollary 5.2. We consider the family

$$
\mathcal{F}=\left\{\left(\mathcal{Y}, y^{\mathrm{c}}\right),\left(\mathcal{V}_{1}, \mathcal{V}_{1}^{\mathrm{c}}\right),\left(\mathcal{T}_{i}, \mathcal{T}_{i}^{\mathrm{c}}\right), y\left(\mathcal{T}_{i}, \mathcal{T}_{i}^{\mathrm{c}}\right) \mid i=1,2\right\}
$$

of walls of $(G, \mathcal{W})$. By Lemma 5.4, for each $i$ the two vertizontal $i$-walls cross. Let us check the other intersections:

- $e \in \mathcal{Y} \cap \mathcal{V}_{1}, t_{1} \in \mathcal{Y} \cap \mathcal{V}_{1}^{\mathrm{c}}, y \in y^{\mathrm{c}} \cap \mathcal{V}_{1}$ and $y t_{1} \in y^{\mathrm{c}} \cap \mathcal{V}_{1}^{\mathrm{c}}$ so that $\left(y, y^{c}\right)$ and $\left(\mathcal{V}_{1}, \mathcal{V}_{1}^{\mathrm{c}}\right)$ cross.

- $e \in \mathcal{T}_{1} \cap \mathcal{T}_{2}, t_{1} \in \mathcal{T}_{1}^{\mathrm{c}} \cap \mathcal{T}_{2}, t_{2} \in \mathcal{T}_{1} \cap \mathcal{T}_{2}^{\mathrm{c}}, t_{2} t_{1} \in \mathcal{T}_{1}^{\mathrm{c}} \cap \mathcal{T}_{2}^{\mathrm{c}}$ so that $\left(\mathcal{T}_{1}, \mathcal{T}_{1}^{\mathrm{c}}\right)$ and $\left(\mathcal{T}_{2}, \mathcal{T}_{2}^{\mathrm{c}}\right)$ cross.

For the intersections $\mathcal{T}_{i}^{\mathrm{c}} \cap y \mathcal{T}_{j}^{\mathrm{c}}$ in the following two items, we refer the reader to Figure 5 (the $t_{i}$-edges in $E_{i}$ are the thick edges, the $t_{j}$-edges in $y E_{j}$ are the dotted edges). 
- $e \in \mathcal{T}_{1} \cap y \mathcal{T}_{2}, t_{1} \in \mathcal{T}_{1}^{\mathrm{c}} \cap y \mathcal{T}_{2}, y t_{2} \in \mathcal{T}_{1} \cap y \mathcal{T}_{2}^{\mathrm{c}}, t_{1} y x_{2}=y x_{1}^{-1} t_{1} x_{2} \in \mathcal{T}_{1}^{\mathrm{c}} \cap y \mathcal{T}_{2}^{\mathrm{c}}$ so that $\left(\mathcal{T}_{1}, \mathcal{T}_{1}^{\mathrm{c}}\right)$ and $y\left(\mathcal{T}_{2}, \widetilde{T}_{2}^{\mathrm{c}}\right)$ cross.

- $e \in y \mathcal{T}_{1} \cap \mathcal{T}_{2}, y t_{1} \in y \mathcal{T}_{1}^{\mathrm{c}} \cap \mathcal{T}_{2}, t_{2} \in y \mathcal{T}_{1} \cap \mathcal{T}_{2}^{\mathrm{c}}, y x_{2}^{-1} t_{2} x_{1}=t_{2} y x_{1} \in y \mathcal{T}_{1}^{\mathrm{c}} \cap \mathcal{T}_{2}^{\mathrm{c}}$ so that $y\left(\mathcal{T}_{1}, \mathcal{T}_{1}^{\mathrm{c}}\right)$ and $\left(\mathcal{T}_{2}, \mathcal{T}_{2}^{\mathrm{c}}\right)$ cross.

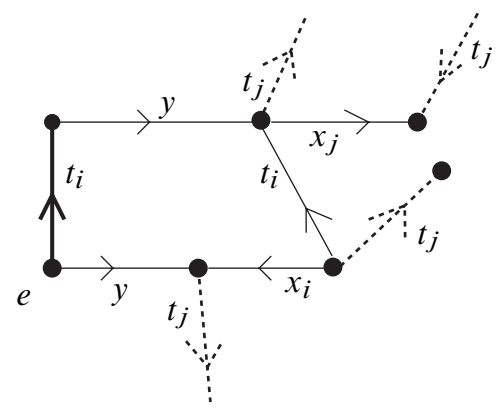

Figure 5

- For each $i \in\{1,2\}, e \in \mathcal{Y}_{\mathcal{T}_{i}}, y \in \mathcal{y}^{\mathrm{c}} \cap \mathcal{T}_{i}, t_{i} \in \mathcal{Y} \cap \mathcal{T}_{i}^{\mathrm{c}}, y t_{i} y^{-1} t_{i} \in y^{\mathrm{c}} \cap \mathcal{T}_{i}^{\mathrm{c}}$ so that $\left(\mathcal{Y}, \mathscr{y}^{\mathrm{c}}\right)$ and $\left(\mathcal{T}_{i}, \mathcal{T}_{i}^{\mathrm{c}}\right)$ cross for each $i \in\{1,2\}$.

- For each $i \in\{1,2\}, e \in y \cap y \mathcal{T}_{i}, y \in y^{\mathrm{c}} \cap y{\widetilde{\tau_{i}}}_{i}, y t_{i} \in y^{\mathrm{c}} \cap y{\widetilde{\mathcal{T}_{i}}}^{\mathrm{c}}, t_{i} y t_{i} \in$ $y \cap y \mathcal{T}_{i}^{\mathrm{c}}$ (since $y t_{i}\left(y^{-1} t_{i} y=t_{i} y x_{i}\left(t_{i} x_{i}^{-1} y^{-1} y\right)=t_{i} y t_{i}\left(x_{i}^{-1} x_{i}\right)\left(y^{-1} y\right)\right)$ so that $\left(\mathcal{Y}, \mathcal{Y}^{\mathrm{c}}\right)$ and $y\left(\mathcal{T}_{i}, \mathcal{T}_{i}^{\mathrm{c}}\right)$ cross for each $i \in\{1,2\}$.

- $e \in \mathcal{V}_{1} \cap \mathcal{T}_{1}, x_{1}=y^{-1} t_{1}^{-1} y t_{1} \in \mathcal{V}_{1} \cap \mathcal{T}_{1}^{\mathrm{c}}, t_{1} \in \mathcal{V}_{1}^{\mathrm{c}} \cap \mathcal{T}_{1}^{\mathrm{c}}, y t_{1} y^{-1}=t_{1} y x_{1} y^{-1} \in$ $\mathcal{V}_{1}^{\mathrm{c}} \cap \mathcal{T}_{1}$ so that $\left(\mathcal{V}_{1}, \mathcal{V}_{1}^{\mathrm{c}}\right)$ and $\left(\mathcal{T}_{1}, \mathcal{T}_{1}^{\mathrm{c}}\right)$ cross.

- $e \in \mathcal{V}_{1} \cap \mathcal{T}_{2}, t_{1} \in \mathcal{V}_{1}^{\mathrm{c}} \cap \mathcal{T}_{2}, t_{2} \in \mathcal{V}_{1} \cap \mathcal{T}_{2}^{\mathrm{c}}, t_{1} t_{2} \in \mathcal{V}_{1}^{\mathrm{c}} \cap \mathcal{T}_{2}^{\mathrm{c}}$ so that $\left(\mathcal{V}_{1}, \mathcal{V}_{1}^{\mathrm{c}}\right)$ and $\left(\mathcal{T}_{2}, \mathcal{T}_{2}^{\mathrm{c}}\right)$ cross.

- $e \in \mathcal{V}_{1} \cap y \mathcal{T}_{1}, t_{1} \in \mathcal{V}_{1}^{\mathrm{c}} \cap y \mathcal{T}_{1}, y t_{1}=t_{1} y x_{1} \in \mathcal{V}_{1}^{\mathrm{c}} \cap y \mathcal{T}_{1}^{\mathrm{c}}, y x_{1}=y\left(y^{-1} t_{1}^{-1} y\right) t_{1} \in$ $\mathcal{V}_{1} \cap y \mathcal{T}_{1}^{\mathrm{c}}$ so that $\left(\mathcal{V}_{1}, \mathcal{V}_{1}^{\mathrm{c}}\right)$ and $y\left(\mathcal{T}_{1}, \mathcal{T}_{1}^{\mathrm{c}}\right)$ cross.

- $e \in \mathcal{V}_{1} \cap y \mathcal{T}_{2}, t_{1} \in \mathcal{V}_{1}^{\mathrm{c}} \cap y \mathcal{T}_{2}, y t_{2} \in \mathcal{V}_{1} \cap y \mathcal{T}_{2}^{\mathrm{c}}, y t_{1} t_{2}=t_{1} y x_{1} t_{2} \in \mathcal{V}_{1}^{\mathrm{c}} \cap y \mathcal{T}_{2}^{\mathrm{c}}$ so that $\left(\mathcal{V}_{1}, \mathcal{V}_{1}^{\mathrm{c}}\right)$ and $y\left(\mathcal{T}_{2}, \mathcal{T}_{2}^{\mathrm{c}}\right)$ cross.

Thus the given family $\mathcal{F}=\left\{\left(\mathcal{Y}, \mathcal{Y}^{\mathrm{c}}\right),\left(\mathcal{V}_{1}, \mathcal{V}_{1}^{\mathrm{c}}\right),\left(\mathcal{T}_{i}, \mathcal{T}_{i}^{\mathrm{c}}\right), y\left(\mathcal{T}_{i}, \mathcal{T}_{i}^{\mathrm{c}}\right) \mid i=1,2\right\}$ is a family of 6 pairwise crossing walls of $(G, \mathcal{W})$. By Lemmas 5.3 and 5.4, in a family of pairwise crossing walls there are at most one horizontal wall, one vertical wall and two vertizontal $i$-walls for each $i \in\{1,2\}$. Therefore such a family contains at most 6 distinct walls. The proof of Corollary 5.2, and so of Theorem 1, at least in the case where $n=2$, is complete.

Remark 5.5. As we noticed the generalization to any integer $n \geq 3$ is straightforward: if $\mathbb{F}_{n}=\left\langle t_{1}, \ldots, t_{n}\right\rangle$ denotes the vertical subgroup of $G=\mathbb{F}_{n+1} \rtimes_{\sigma} \mathbb{F}_{n}$, then as above the vertical walls are the classical walls of the free group $\mathbb{F}_{n}$; if $\mathbb{F}_{n+1}=$ $\left\langle x_{1}, \ldots, x_{n}, y\right\rangle$ denotes the horizontal subgroup of $G$, then as above the horizontal walls are the left $G$-translates of the wall separated by the edges in $\bigcup_{t \in \mathbb{F}_{n}}(t, t y)^{ \pm 1}$. 
Finally there is a type of vertizontal wall for each letter in $\left\{t_{1}, \ldots, t_{n}\right\}$ and $i$-vertizontal walls are defined as in 3.1 by posing $H_{i}=\left\langle x_{j}, t_{j}, y x_{i} y^{-1} t_{i}, x_{i} t_{i}^{-1} \mid j \neq i\right\rangle$. The dimension of the cube complex on which $G$ acts is $2 n+2$ : the +2 comes from the fact that one can always put one horizontal and one vertical wall in a family of pairwise crossing walls, and no more. The $2 n$ comes from the fact that there are $n$ distinct types of vertizontal walls and for each vertizontal $i$-wall one can put two distinct $i$-walls in a family of pairwise crossing walls, and no more.

Remark 5.6. By adapting our construction we get that the group $\mathbb{F}_{n+1} \rtimes_{\sigma} \mathbb{F}_{2}(n \geq 3)$, where $\sigma\left(t_{i}\right)$ fixes any $x_{j}$ for $j=1, \ldots, n$ and $\sigma\left(t_{i}\right)(y)=y x_{i}, i=1,2$, acts on a 6dimensional CAT $(0)$ cube complex: the walls are the vertical walls defined above, the vertizontal walls associated to $t_{i}$ defined in a similar way as above (in the definition of the subgroup $H_{i}$ add $x_{3}, \ldots, x_{n}$ as generators) and horizontal walls associated not only to $y$ but also to $x_{3}, \ldots, x_{n}$. There are more types of horizontal walls but less types of vertizontal walls than in the $n^{\text {th }}$-group of Formanek-Procesi. Since two distinct horizontal walls cannot be in a collection of pairwise crossing walls (contrary to what happens with vertizontal walls), this explains the smaller dimension of the complex in this case. Thus what is perhaps the most important, for the dimension of the cube complex, is the way the images of the higher edges cover the lower strata. Here the rose with $n+1$ petals is a Bestvina-Feighn-Handel representative. The filtration of the graph is given by $\emptyset \varsubsetneqq\left\{x_{1}, \ldots, x_{n}\right\} \varsubsetneqq\left\{x_{1}, \ldots, x_{n}\right\} \cup\{y\}$. The image of the highest edge $y$ cover $\left\{x_{1}, x_{2}\right\}$ but not the whole lower stratum $\left\{x_{1}, \ldots, x_{n}\right\}$ as it is the case when considering the $n^{\text {th }}$-group of Formanek-Procesi.

Acknowledgements. The author would like to thank M. Lustig (Université d'AixMarseille III, Marseille) for giving to him, while working on other topics, the example of free-by-free group we deal with in this paper. It is a pleasure to thank P. A. Cherix (Université de Genève, Geneva) who introduced the author to the Haagerup property seven years ago. G. N. Arzhantseva (Universität Wien, Vienna) and P. A. Cherix evoked the question of the a-T-menability of free-by-free, and surface-by-free groups. At that time in Geneva, the interest of W. Pitsch (U.A.B., Barcelona) in this question was a source of motivation. For these reasons, all three of them deserve the gratitude of the author. He is moreover indebted to G. N. Arzhantseva for telling him about the Question cited in the Introduction. A part of these acknowledgements also goes to G. N. Arzhantseva, M. Lustig and Y. Stalder for their remarks about preliminary versions of the paper. Last but not least, many thanks are due to the referee, who provided a great help to correct some mistakes and gave invaluable comments to make the paper better: among many other things he indicated to the author the results of Chatterji-Niblo [5] and Nica [16], and gave him the idea of looking for the dimension of the cube complex. 


\section{References}

[1] C. A. Akemann and M. E. Walter, Unbounded negative definite functions. Canad. J. Math. 33 (1981), 862-871. Zbl 0437.22004 MR 634144

[2] M. Bestvina, M. Feighn, and M. Handel, The Tits alternative for $\operatorname{Out}\left(F_{n}\right)$ II: A Kolchin type theorem. Ann. of Math. (2) 161 (2005), 1-59. Zbl 1139.20026 MR 2150382

[3] M. R. Bridson and A. Haefliger, Metric spaces of non-positive curvature. Grundlehren Math. Wiss. 319, Springer-Verlag, Berlin 1999. Zbl 0988.53001 MR 1744486

[4] M. Burger, Kazhdan constants for SL(3, Z). J. Reine Angew. Math. 413 (1991), 36-67. Zbl 0704.22009 MR 1089795

[5] I. Chatterji and G. Niblo, From wall spaces to CAT(0) cube complexes. Internat. J. Algebra Comput. 15 (2005), 875-885. Zbl 1107.20027 MR 2197811

[6] P.-A. Cherix, M. Cowling, P. Jolissaint, P. Julg, and A. Valette, Groups with the Haagerup property. Progr. Math. 197, Birkhäuser, Basel 2001. Zbl 1030.43002 MR 1852148

[7] Y. de Cornulier, Y. Stalder, and A. Valette, Proper actions of lamplighter groups associated with free groups. C. R. Math. Acad. Sci. Paris 346 (2008), 173-176. Zbl 1132.43001 MR 2393636

[8] Y. Cornulier, Y. Stalder, and A. Valette, Proper actions of wreath products and generalizations. Trans. Amer. Math. Soc. 364 (2012), 3159-3184. Zbl 06038811 MR 2888241

[9] P. de la Harpe and A. Valette, La propriété (T) de Kazhdan pour les groupes localement compacts. Astérisque 175 (1989). Zbl 0759.22001 MR 1023471

[10] E. Formanek and C. Procesi, The automorphism group of a free group is not linear. $J$. Algebra 149 (1992), 494-499. Zbl 0780.20023 MR 1172442

[11] E. Guentner and N. Higson, Weak amenability of CAT(0)-cubical groups. Geom. Dedicata 148 (2010), 137-156. Zbl 1208.46054 MR 2721622

[12] U. Haagerup, An example of a nonnuclear $C^{*}$-algebra, which has the metric approximation property. Invent. Math. 50 (1979), 279-293. Zbl 0408.46046 MR 520930

[13] F. Haglund and F. Paulin, Simplicité de groupes d'automorphismes d'espaces à courbure négative. In The Epstein Birthday Schrift, Geom. Topol. Monogr. 1, Geom. Topol. Publ., Coventry 1998, 181-248. Zbl 0916.51019 MR 1668359

[14] P. Jolissaint, Borel cocycles, approximation properties and relative property T. Ergodic Theory Dynam. Systems 20 (2000), 483-499. Zbl 0955.22008 MR 1756981

[15] G. A. Niblo and M. A. Roller, Groups acting on cubes and Kazhdan's property (T). Proc. Amer. Math. Soc. 126 (1998), 693-699. Zbl 0906.20024 MR 1459140

[16] B. Nica, Cubulating spaces with walls. Algebr. Geom. Topol. 4 (2004), 297-309. Zbl 1131.20030 MR 2059193

[17] M. Sageev, Ends of group pairs and non-positively curved cube complexes. Proc. London Math. Soc. (3) 71 (1995), 585-617. Zbl 0861.20041 MR 1347406

Received November 27, 2009; revised June 19, 2011

F. Gautero, Université de Nice Sophia Antipolis, Laboratoire de Mathématiques

J. A. Dieudonné (UMR CNRS 7351), Parc Valrose, 06108 Nice Cedex 2, France

E-mail: Francois.Gautero@unice.fr 